\title{
DYNAMIC MODE DECOMPOSITION FOR CONSTRUCTION OF REDUCED-ORDER MODELS OF HYPERBOLIC PROBLEMS WITH SHOCKS
}

\author{
Hannah Lu E Daniel M. Tartakovsky* \\ Department of Energy Resources Engineering, Stanford University, Stanford, \\ CA 94305, USA \\ *Address all correspondence to: Daniel M. Tartakovsky, Department of Energy Resources \\ Engineering, Stanford University, Stanford, CA 94305, USA, \\ E-mail: tartakovsky@stanford.edu
}

Original Manuscript Submitted: 9/8/2020; Final Draft Received: 12/28/2020

Construction of reduced-order models (ROMs) for hyperbolic conservation laws is notoriously challenging mainly due to the translational property and nonlinearity of the governing equations. While the Lagrangian framework for ROM construction resolves the translational issue, it is valid only before a shock forms. Once that occurs, characteristic lines cross each other and projection from a highfidelity model space onto a ROM space distorts a moving grid, resulting in numerical instabilities. We address this grid distortion issue by developing a physics-aware dynamic mode decomposition (DMD) method based on hodograph transformation. The latter provides a map between the original nonlinear system and its linear counterpart, which coincides with the Koopman operator. This strategy is consistent with the spirit of physics-aware DMDs in that it retains information about shock dynamics. Several numerical examples are presented to validate the proposed physics-aware DMD approach for construction of accurate ROMs.

KEY WORDS: conservation law, Koopman operator, proper orthogonal decomposition, hodograph transformation, learning ROMs

\section{INTRODUCTION}

Since introduction of Euler equations, hyperbolic conservation laws play a significant role in gas dynamics, astrophysics, plasma, traffic flow, multiphase flow in porous media (Bear, 2013; Chang and Hsiao, 1989; Courant and Friedrichs, 1999; Shu, 1991; Whitham, 2011) and other fields of science and engineering. Wave-like solutions of hyperbolic equations can exhibit various rarefaction and shock behaviors, whose occurrence strongly depends on a functional form of the flux function. Discontinuity and uniqueness of such solutions pose challenges in theoretical treatment of hyperbolic conservation laws (Oleinik, 1957; Smoller, 2012). Theoretical advances, such as entropy conditions and the concept of a weak solution (Harten, 1983; Lax, 1971), ameliorate this difficulty by providing physical interpretation to these solutions. Likewise, numerical high-resolution methods have been designed to resolve nonlinearities and accurately capture shocks (LeVeque, 1992; Majda and Osher, 1979; Osher and Chakravarthy, 1984). Although continued developments in scientific computing have improved the performance of 
high-resolution simulations, their computational cost is often too high to model complex systems at spatiotemporal resolutions and scales of interest. The cost can become prohibitive when used in the context of uncertainty quantification or data assimilation, both of which require a large number of repeated forward model runs.

Reduced-order models (ROMs) provide an efficient alternative to their high-fidelity, physicsbased counterparts that can be deployed in large-scale multiphysics simulations. Robust tools for construction of ROMs for problems described by ordinary differential equations or parabolic partial differential equations (PDEs) include proper orthogonal decomposition (POD) (Benner et al., 2015; Kerschen et al., 2005; Rowley, 2005) and dynamic mode decomposition (DMD) (Alessandro and Nathan, 2017; Brunton et al., 2016; Schmid, 2010; Williams et al., 2013). The challenge of extending these techniques to hyperbolic or advection-dominated parabolic PDEs with smooth solutions was met by Lu and Tartakovsky (2020a) through development of the physics-aware DMD and POD approaches within a Lagrangian framework. However, in the presence of strong shocks and/or sharp gradients, Lagrangian POD methods can generate numerical instability caused by grid distortion (Mojgani and Balajewicz, 2017). Once characteristics of a nonlinear hyperbolic PDE intersect each other, the projection from a high-dimensional manifold of the high-fidelity model (HFM) onto a low-dimensional subspace of the low-fidelity model (e.g., ROM) is not guaranteed and typically fails to preserve topological properties of the original HFM. We elaborate on this point in Section 2, in terms relevant to DMD.

We use hodograph transformation (Hamilton, 1847) to resolve this outstanding issue in construction of ROMs for PDEs with discontinuous solutions and shocks. Hodograph diagrams originated in meteorology to plot wind from soundings of Earth's atmosphere. Since then, hodograph transformation morphed into a technique designed to transform nonlinear PDEs into linear ones by interchanging the dependent and independent variables. Hodograph-type transformations have been used to find quasilinear analogs of semilinear equations and to derive new analytical solutions to special classes of PDEs (Clarkson et al., 1989). Advantages of mapping nonlinear PDEs onto their linear counterparts are self-evident: analytical tools available for linear PDEs provide better understanding of the behavior of a solution, and numerical solvers for linear systems are both easier to implement and computationally cheaper.

The Koopman operator theory (Koopman, 1931) shares the goal of hodograph transformation: a Koopman operator is an infinite-dimensional linear operator that represents the underlying finite-dimensional nonlinear dynamic system by judiciously choosing observable functions. It is also similar in its goal to integral transformations that map certain classes of nonlinear PDEs onto their linear counterparts; for example, the Cole-Hopf transformation and the Kirchhoff transformation map, respectively, Burgers' equation and a class of nonlinear diffusion (heat conduction) equations onto a linear diffusion equation. These integral transformations have been used in the context of the Koopman operator theory and DMD/POD to constructed ROMs for Burgers' equation (Kutz et al., 2018) and a nonlinear diffusion equation (Lu and Tartakovsky, 2020b). A major goal of our study is to establish clear connections between hodograph transformation and the Koopman theory. This relationship between the two is then used both to identify observables for a Koopman operator via hodograph transformation and to construct ROMs for hyperbolic conservation laws with shocks via DMD.

Besides the choice of the observable functions, another key ingredient of the success of a DMD algorithm is data availability. As proved theoretically by $\mathrm{Tu}$ et al. (2014) and verified numerically by Lu and Tartakovsky (2020b), data have to be sufficiently rich for the learning algorithm to capture all essential features of the underlying dynamics. Therefore, a key condition in our DMD framework for mixed wave problems is that the data be collected until and after all 
forms of propagation occurs. A resulting ROM remains valuable as a predictor of the continuing propagation. For example, the Buckley-Leverett equation (see Section 5.5) is widely used in the oil and gas industries to describe water injection and oil production processes. A shock profile forms right after the injection begins. Quantities of interest are breakthrough time (i.e., the time when the shock front exits the domain) and water-cut curve (i.e., the cumulative rarefaction curve after the breakthrough), which can be efficiently predicted by a successful ROM.

A general procedure of the physics-aware DMD algorithm and its connection with Koopman operators are reviewed in Section 2. This section also contains a numerical demonstration of the failure of Lagrangian-based ROMs to capture the dynamics described by conservation laws with shocks. We illustrate the use of hodograph transformation by analyzing the inviscid Burgers' equation (Section 3) and more general hyperbolic PDEs with a convex flux function (Appendix A). In Section 4, we combine hodograph transformation with the Koopman operator theory to design a physics-aware DMD algorithm for construction of ROMs for conservation laws with shocks. Several numerical tests are presented in Section 5 to validate the proposed physics-aware DMD approach. Main conclusions drawn from our study are summarized in Section 6.

\section{CONSTRUCTION OF ROMS AND THEIR FAILURE FOR PROBLEMS WITH SHOCKS}

Consider a state variable $u(x, t):[a, b] \times[0, T] \rightarrow \mathbb{R}$, where the constants $a, b \in \mathbb{R}$ and $T \in \mathbb{R}^{+}$. The dynamics of $u(x, t)$ is described by a one-dimensional scalar conservation law:

$$
\frac{\partial u}{\partial t}+\frac{\partial F(x, t, u)}{\partial x}=0 \quad \text { or } \quad \frac{\partial u}{\partial t}+f(x, t, u) \frac{\partial u}{\partial x}=0, \quad f(x, t, u)=\frac{\partial F(x, t, u)}{\partial u} .
$$

This hyperbolic PDE is subject to the initial condition $u(x, t=0)=u_{0}(x)$ and, when appropriate (i.e., when $|a|,|b|<\infty$ ), boundary conditions at $a$ and/or $b$. The intervals $[0, T]$ and $[a, b]$ are discretized with $(N+1)$ and $J_{x}$ nodes separated, respectively, by $\Delta t$ and $\Delta x$. The uniform spacial mesh is denoted by $\tilde{\mathbf{x}}=\left[x_{1}, \ldots, x_{J_{x}}\right]^{\top}$. To be specific, we solve Eq. (2.1) with a conservative first-order upwind scheme (LeVeque, 1992):

$$
u_{j}^{n+1}=u_{j}^{n}-\frac{\Delta t}{\Delta x}\left(F_{j+1 / 2}^{n}-F_{j-1 / 2}^{n}\right),
$$

where $n=0, \ldots, N$ indicates the $n$th time step, with $n=0$ corresponding to $t=0$, and $n=N$ to $t=T ; j=1, \ldots, J_{x}$ denotes the $j$ th spatial node, such that $j=1$ and $J_{x}$ coincide with $x=a$ and $b$, respectively; and

$$
\begin{aligned}
& F_{j+1 / 2}^{n}=\frac{F\left(\cdot, u_{j+1}^{n}\right)+F\left(\cdot, u_{j}^{n}\right)}{2}-\left|\alpha_{j+1 / 2}^{n}\right| \frac{u_{j+1}^{n}-u_{j}^{n}}{2}, \\
& \alpha_{j+1 / 2}^{n}= \begin{cases}\frac{F_{j+1}^{n}-F_{j}^{n}}{u_{j+1}^{n}-u_{j}^{n}} & \text { if } \quad u_{j+1}^{n} \neq u_{j}^{n}, \\
f\left(\cdot, u_{j}\right) & \text { if } \quad u_{j+1}^{n}=u_{j}^{n} .\end{cases}
\end{aligned}
$$

A numerical solution provided by Eq. (2.2) with sufficiently small $\Delta t$ and $\Delta x$, satisfying the corresponding Courant-Friedrichs-Lewy (CFL) condition, are referred to as a reference HFM throughout the paper.

Volume 2, Issue 1, 2021 
Standard (Eulerian) approaches to construction of a ROM for Eq. (2.1) often fail due to the traveling-wave nature of its solution (Lu and Tartakovsky, 2020a; Mojgani and Balajewicz, 2017). In a shock-free scenario, the Lagrangian framework can resolve the translational issue in the POD or DMD approaches to ROMs by keeping track of the characteristic lines.

In the Lagrangian framework, Eq. (2.1) becomes

$$
\left\{\begin{array}{l}
\frac{\mathrm{d} x}{\mathrm{~d} t}=f(x, t, u), \quad x(0)=\eta, \\
\frac{\mathrm{d} u}{\mathrm{~d} t}=0, \quad u(\eta, 0)=u_{0}(\eta),
\end{array}\right.
$$

where $\eta \in \mathbb{R}$ is a label of the characteristic $x(t)$. As in the Eulerian case, we use the uniform discretization of the time interval $[0, T]$, such that $0=t^{0}<t^{1}<\ldots<t^{N}=T$ with time step $\Delta t=t^{n+1}-t^{n}$. At time $t=0$, the space, $[a, b]$, is discretized with a uniform mesh $\mathbf{x}^{0}=\left[x_{1}^{0}, \ldots, x_{J_{x}}^{0}\right]^{\top}$ of mesh size $\Delta x^{0}=x_{j+1}^{0}-x_{j}^{0}$. Unlike its Eulerian counterpart, the spatiotemporal discretization of $u(x, t)$ in the Lagrangian framework, $\mathbf{u}^{n}=\left[u_{1}^{n}, \ldots, u_{J_{x}}^{n}\right]^{\top}$ for $n=0, \ldots, N$, may be nonuniform in space due to the temporal evolution of the grid nodes $x_{j}(t)$. The backward Euler discretization, used by Mojgani and Balajewicz (2017), transforms Eq. (2.3) into

$$
\left\{\begin{array}{l}
x_{j}^{n+1}=x_{j}^{n}+\Delta t f\left(x_{j}^{n+1},(n+1) \Delta t, u_{j}^{n+1}\right), \\
u_{j}^{n+1}=u_{j}^{n}
\end{array}\right.
$$

or, in vector form,

$$
\left\{\begin{array}{l}
\mathbf{R}_{x}\left(\mathbf{x}^{n+1}\right) \equiv \mathbf{x}^{n+1}-\mathbf{x}^{n}-\Delta t \mathbf{f}^{n+1}\left(\cdot, \mathbf{u}^{n+1}\right)=0 \\
\mathbf{R}_{u}\left(\mathbf{u}^{n+1}\right) \equiv \mathbf{u}^{n+1}-\mathbf{u}^{n}=0
\end{array}\right.
$$

where $\mathbf{x}^{n}=\left[x_{1}^{n}, \ldots, x_{J_{x}}^{n}\right]^{\top}$ denotes the nodes of the Lagrangian computational grid at the $n$th time step. This numerical scheme involves $N$ iterations in the two high-dimensional $J_{x} \times 1$ vectors, $\mathbf{x}^{n+1}$ and $\mathbf{u}^{n+1}$. It provides a Lagrangian HFM.

To construct a ROM, a data set consisting of a sequence of $M$ solution snapshots $(M \leq N$ and, ideally, $M \ll N)$ is collected from the HFM. Since $\mathbf{u}^{n}$ is conservative and invariant in time, we only need the data matrix $\mathbf{X}$ with $M$ snapshots of the Lagrangian grid $\mathbf{x}^{n}$ :

$$
\mathbf{X}=\left[\begin{array}{cccc}
\mid & \mid & & \mid \\
\mathbf{x}^{1} & \mathbf{x}^{2} & \ldots & \mathbf{x}^{M} \\
\mid & \mid & & \mid
\end{array}\right]
$$

In the next two subsections, we briefly revisit the algorithms of Lagrangian POD (Mojgani and Balajewicz, 2017) and Lagrangian DMD (Lu and Tartakovsky, 2020a) used to construct a ROM.

\subsection{Lagrangian POD}

Identification of the POD modes is based on a reduced singular value decomposition (SVD),

$$
\mathbf{X}=\mathbf{U} \boldsymbol{\Sigma} \mathbf{V}^{*}
$$

where $\mathbf{U} \in \mathbb{C}^{J_{x} \times K}$ and $\mathbf{V} \in \mathbb{C}^{M \times K}$ are orthogonal matrices, $\boldsymbol{\Sigma}=\mathbb{C}^{K \times K}$ is a diagonal matrix, and $K$ is the rank of the matrix $\mathbf{X}$ approximated by the reduced SVD. Further rank truncation can be achieved by using the energy criterion, 


$$
r=\min _{k}\left\{\frac{\sigma_{k}}{\sum_{k^{\prime}=1}^{K} \sigma_{k^{\prime}}}<\varepsilon\right\},
$$

where $\sigma_{k}$ are the diagonal elements of $\boldsymbol{\Sigma}$, and $\varepsilon$ is a small number (tolerance), chosen to be $\varepsilon=10^{-4}$ in all our numerical examples. After the truncation, we get the POD modes

$$
\boldsymbol{\Phi}=\mathbf{U}(:, 1: r)=\left[\begin{array}{cccc}
\mid & \mid & & \mid \\
\phi_{1} & \phi_{2} & \ldots & \phi_{r} \\
\mid & \mid & & \mid
\end{array}\right] .
$$

Notice that $r \ll K \leq \min \left\{J_{x}, M\right\}$, and the basis $\left\{\phi_{1}, \ldots, \phi_{r}\right\}$ is orthonormal. Galerkin projection in the low-dimensional space spanned by the POD basis provides a ROM (low-fidelity solution),

$$
\mathbf{x}_{\mathrm{POD}}^{n+1}=\sum_{k=1}^{r} \hat{x}_{k}^{n+1} \phi_{k}=\mathbf{\Phi} \hat{\mathbf{x}}^{n+1} .
$$

The $r \times 1$ vector $\hat{\mathbf{x}}^{n+1}$ of coefficients is computed as a solution of

$$
\boldsymbol{\Phi}^{\top} \mathbf{R}\left(\boldsymbol{\Phi}\left[\begin{array}{c}
\mid \\
\hat{\mathbf{x}}^{n+1} \\
\mid
\end{array}\right]\right)=0,
$$

that is obtained by substituting Eq. (2.10) into the first equation in Eq. (2.5) and projecting onto the subspace spanned by $\boldsymbol{\Phi}$.

\subsection{Lagrangian DMD}

Lagrangian DMD (Lu and Tartakovsky, 2020a) applies the standard DMD to the Lagrangian grid matrix $\mathbf{X}$ in Eq. (2.6). We denote by $\mathbf{K}$ the evolution operator of the characteristic lines $\mathbf{x}^{n}$ described in Eq. (2.4); that is

$$
\mathbf{x}^{n+1}=\mathbf{K} \mathbf{x}^{n},
$$

with initial uniform mesh $\mathbf{x}^{0}$. Evolution of the Lagrangian mesh grid is predicted by the Algorithm 1, and solution $\mathbf{u}^{n}$ can be interpolated afterward.

\subsection{ROM Failure for Problems with Shocks: Inviscid Burgers' Equation}

One of the most studied examples of Eq. (2.1) is the inviscid Burgers' equation:

$$
\frac{\partial u}{\partial t}+u \frac{\partial u}{\partial x}=0, \quad u(x, 0)=u_{0}(x)
$$

which we define on the space-time domain $(x, t) \in[0,2 \pi] \times[0,1]$. Depending on the boundary and initial conditions, this problem admits both smooth and discontinuous solutions $u(x, t)$. For example, a smooth solution is obtained for the periodic boundary conditions, $u(0, t)=u(2 \pi, t)$, and the initial data $u_{0}(x)=1+\sin (x)$. In this setting, standard (Eulerian) ROMs fail due to the inability of SVD to represent a low-rank structure of translational problem, while the ROMs based on either Lagrangian POD or Lagrangian DMD perform well in terms of both accuracy and computational efficiency (Lu and Tartakovsky, 2020a).

Volume 2, Issue 1, 2021 
Algorithm 1: Lagrangian DMD algorithm (Lu and Tartakovsky, 2020a)

0. Create data matrices of $(M-1)$ observables, $\mathbf{X}_{1}$ and $\mathbf{X}_{2}$,

$$
\mathbf{X}_{1}=\left[\begin{array}{cccc}
\mid & \mid & & \mid \\
\mathbf{x}^{1} & \mathbf{x}^{2} & \ldots & \mathbf{x}^{M-1} \\
\mid & \mid & & \mid
\end{array}\right], \quad \mathbf{X}_{2}=\left[\begin{array}{cccc}
\mid & \mid & & \mid \\
\mathbf{x}^{2} & \mathbf{x}^{3} & \ldots & \mathbf{x}^{M} \\
\mid & \mid & & \mid
\end{array}\right] .
$$

1. Apply SVD of matrix $\mathbf{X}_{1} \approx \mathbf{U} \boldsymbol{\Sigma} \mathbf{V}^{*}$ with $\mathbf{U} \in \mathbb{C}^{J_{x} \times r}, \boldsymbol{\Sigma} \in \mathbb{C}^{r \times r}, \mathbf{V} \in \mathbb{C}^{(M-1) \times r}$, where $\mathbf{U}$ and $\mathbf{V}$ are orthogonal matrices, $\boldsymbol{\Sigma}$ is a diagonal matrix, and $r$ is the truncated rank chosen by a certain criterion; for example, Eq. (2.8).

2. Compute $\tilde{\mathbf{K}}=\mathbf{U}^{*} \mathbf{X}_{2} \mathbf{V} \boldsymbol{\Sigma}^{-1}$ as an $r \times r$ low-rank approximation of $\mathbf{K}$.

3. Compute eigen-decomposition of $\tilde{\mathbf{K}}: \tilde{\mathbf{K}} \mathbf{W}=\mathbf{W} \boldsymbol{\Lambda}, \boldsymbol{\Lambda}=\left(\lambda_{k}\right)$.

4. Reconstruct eigen-decomposition of $\mathbf{K}$. Eigenvalues are $\boldsymbol{\Lambda}$ and eigenvectors are $\mathbf{\Phi}=\mathbf{U W}$.

5. Future $x_{D M D}^{n+1}$ is predicted by

$$
\mathbf{x}_{\mathrm{DMD}}^{n+1}=\mathbf{\Phi} \boldsymbol{\Lambda}^{n+1} \mathbf{b}, \quad n>M
$$

with $\mathbf{b}=\boldsymbol{\Phi}^{-1} \mathbf{x}_{1}$.

6. Interpret the solution in the moving grid:

$$
u_{\mathrm{DMD}}\left(x_{j}^{n}, n \Delta t\right)=u_{0}\left(x_{j}^{0}\right) .
$$

A solution to Eq. (2.13) develops shocks in finite time, for example, for a Gaussian-type initial data,

$$
u_{0}(x)=0.8+0.5 \exp \left[-\frac{(x-0.3)^{2}}{0.001}\right] .
$$

In the pure Lagrangian approach [Eq. (2.3)], the discretization has to account for shock formation. Once the characteristic lines cross each other, the Lagrangian mesh becomes sensitive to the choice of discretization of $u[x(t), t]$. For instance, a discretization of Eq. (2.3) with $f(\cdot, u)=u$,

$$
\left\{\begin{array}{l}
u_{j}^{n+1}=u_{j}^{n}, \\
x_{j}^{n+1}=x_{j}^{n}+\Delta t u_{j}^{n+1},
\end{array}\right.
$$

would lead to the so-called overshoot that admits multi-value solutions [Fig. 1(a)], which contradicts the entropy condition. This is a typical problem with the Lagrangian framework. It should come as no surprise that an attempt to build a ROM with the Lagrangian DMD based on the faulty discretization of Eq. (2.18) likewise results in failure [Fig. 1(b)]. The Lagrangian DMD faithfully reproduces the unphysical solution obtained with the faulty discretization scheme [Eq. (2.18)]. In other words, the resulting unphysical ROM is not caused by the DMD algorithm itself; the data from the full Lagrangian model [Eq. (2.18)] provide inaccurate and incomplete (without shock) information from the very beginning.

We consider a numerical scheme that is known for its ability to handle shocks: the backward semi-Lagrangian method 

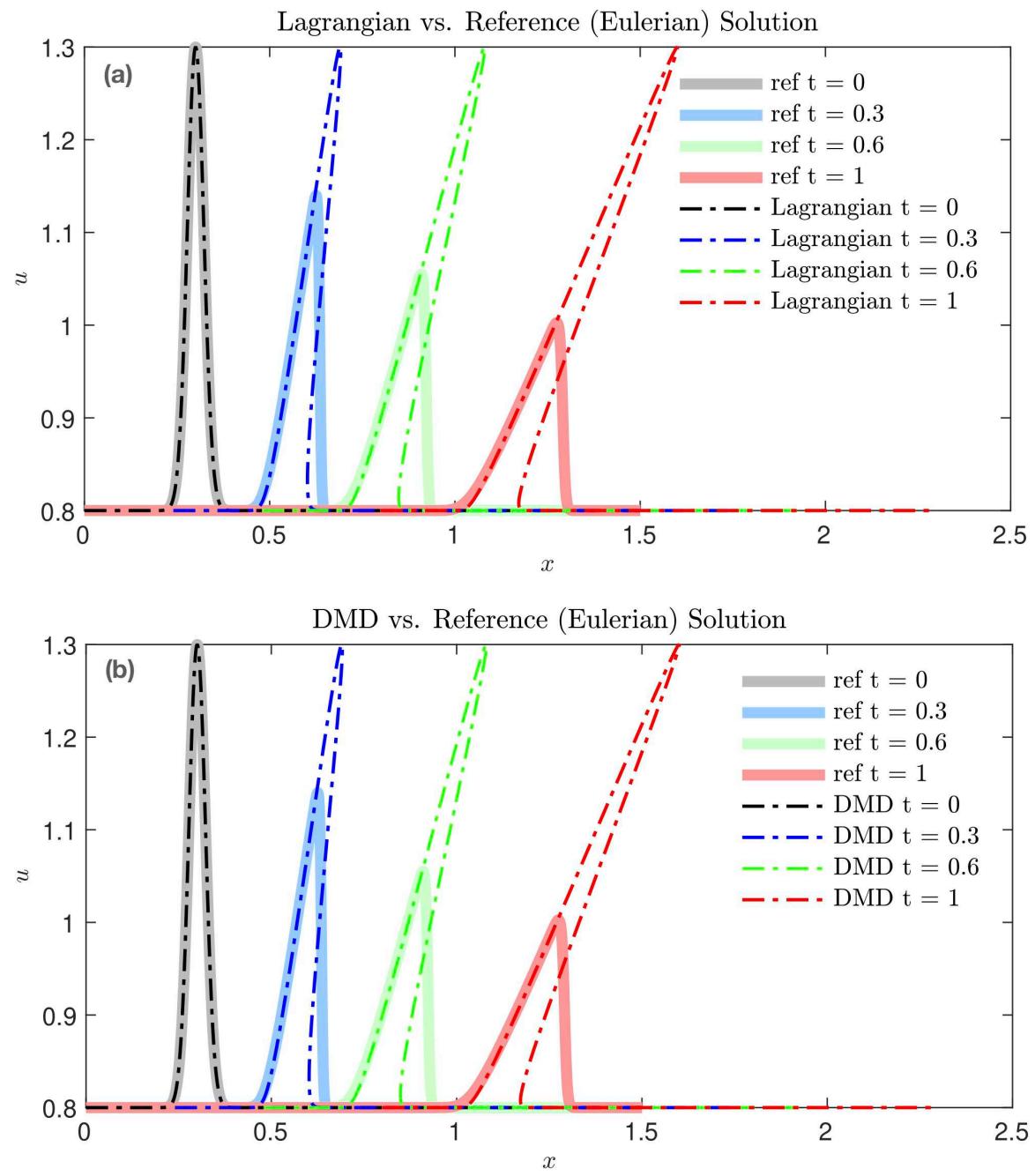

FIG. 1: Solutions of the inviscid Burgers' equation with a shock. (a) The full solution obtained with the Lagrangian numerical scheme [Eq. (2.18)] leading to the overshoot. (b) The Lagrangian DMD solution trained on a few snapshots of the faulty full solution. The reference solution is obtained with Eq. (2.2).

$$
\left\{\begin{array}{l}
u_{j}^{n+1}=u_{j}^{n} \\
x^{*}=x_{j}^{n}+\frac{\Delta t}{2} u_{j}^{n} \\
x_{j}^{n+1}=x_{j}^{n}+\frac{\Delta t}{2}\left[u\left(x^{*}, t^{n}\right)+u\left(x^{*}, t^{n+1}\right)\right]
\end{array}\right.
$$

and employ the (explicit) mid-point rule to avoid implicit iterations. Since $u\left(\cdot, t^{n+1}\right)$ is unchanged from $u\left(\cdot, t^{n}\right)$, the evaluations at $x^{*}$ can be calculated via interpolation (e.g., via linear interpolation used below). Figure 2(a) reveals that this numerical scheme is indeed capable to accurately approximate the solution of the inviscid Burgers' equation with shocks. However, the Lagrangian DMD algorithm using snapshots from the full solution [Eq. (2.19)] suffers from

Volume 2, Issue 1, 2021 

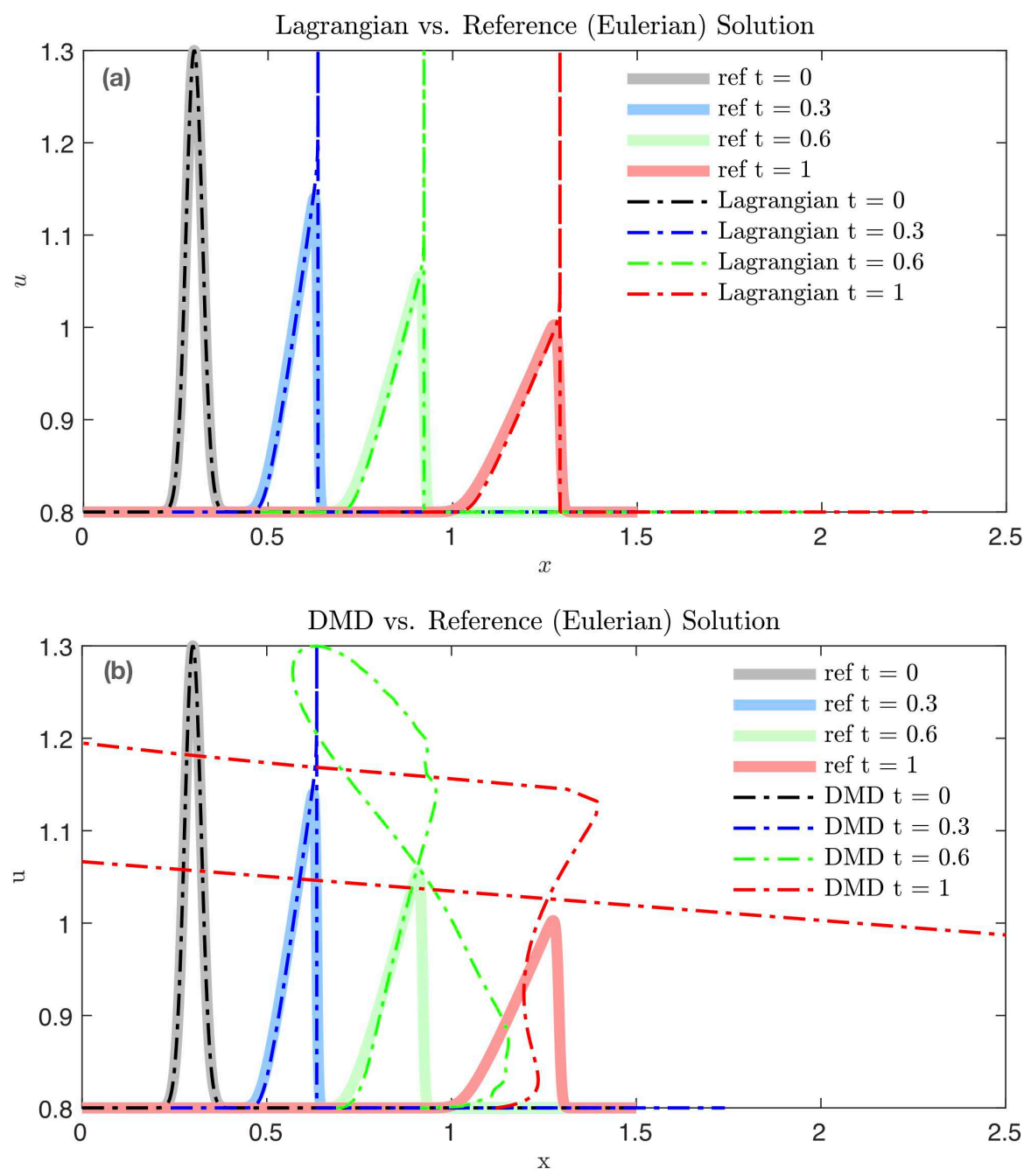

FIG. 2: Solutions of the inviscid Burgers' equation with a shock. (a) The full solution obtained with the appropriate Lagrangian numerical scheme [Eq. (2.19)]. (b) The Lagrangian DMD solution trained on a few snapshots of the accurate full solution. The reference solution is obtained with Eq. (2.2).

instability once a shock is about to form [Fig. 2(b)]. The grid becomes severely distorted once the characteristic lines intersect each other at the interface where sharp gradients of $u(x, t)$ occur. At the intersect, one arrival location of $x$ corresponds to two different departure values of $u$. However, the DMD modes projection from the HFM to the ROM does not keep the topological information about this multivalued mapping in the ROM process, resulting in the Lagrangian grid distortion.

Remark 2.1. The Lagrangian POD approach suffers from similar problems (Mojgani and Balajewicz, 2017). Moreover, the POD projection on the accurate Lagrangian scheme [Eq. 2.19] would still require interpolation in the high-dimensional space. We might need techniques such 
as DEIM (Chaturantabut and Sorensen, 2010) to keep the resulting ROM's efficiency. Nevertheless, extensions of POD are beyond the scope of our study; we focus on DMD-based ROMs due to their iteration-free nature.

\section{HODOGRAPH TRANSFORMATION}

We start with a mathematical definition of hodograph transformation reproduced from Clarkson et al. (1989).

Definition 3.1. A pure hodograph transform is a transformation of the form

$$
\tau=t, \quad \xi=u(x, t) .
$$

For the inviscid Burgers' equation [Eq. (2.13)], we first consider a scenario where only one shock is developed from the initial data $u_{0}(x)$ in finite time. This necessitates the following assumption.

Assumption 3.1. The function $u_{0}(x)$ satisfies four conditions:

- $u_{0}(x)$ is smooth.

- $u_{0}(x)$ decreases monotonically: $u_{0}^{\prime}(x)<0$ for all $x$; and $\lim _{x \rightarrow+\infty} u_{0}(x)=u_{R}$, $\lim _{x \rightarrow-\infty} u_{0}(x)=u_{L}$ with constants $u_{R}<u_{L}$.

- $u_{0}(x)$ has a unique inflection point $\left(x^{*}, u^{*}\right)$ with $u^{*}=u_{0}\left(x^{*}\right)$, meaning $u_{0}^{\prime \prime}\left(x^{*}\right)=0$.

- $u_{0}^{\prime \prime \prime}\left(x^{*}\right)>0$.

This assumption ensures existence of an inverse function $x(t, u):[0, T] \times\left[u_{R}, u_{L}\right] \rightarrow[a, b]$ of the monotonic function $u(t, x):[0, T] \times[a, b] \rightarrow\left[u_{R}, u_{L}\right]$. The last two assumptions ensure single-shock formation for illustration purposes. It follows from Definition 3.1 that the inverse function $x(t, u)=x(\tau, \xi)$ is a pure hodograph transform based on Eq. (3.1).

\subsection{Solution before Shock Formation}

With $u$ acting as the independent variable and $x$ as the dependent variable, hodograph transformation $x=x(t, u)$ maps the equation for characteristics [Eq. (2.3)] of the inviscid Burgers' equation [Eq. (2.13)], before the shock formation time $t^{*}$ (defined later), onto

$$
\frac{\mathrm{d} x(t, u)}{\mathrm{d} t}=u, \quad x(0, u)=x_{0}(u), \quad \text { for } \quad(t, u) \in\left[0, t^{*}\right) \times\left[u_{R}, u_{L}\right] .
$$

Assumption 3.1 translates into conditions on the function $x_{0}(u)$ :

- $x_{0}^{\prime}(u)<0$.

- $x_{0}(u)$ has a unique inflection point at $\left(u^{*}, x^{*}\right)$.

- $x_{0}^{\prime \prime \prime}\left(u^{*}\right)<0$.

Volume 2, Issue 1, 2021 
Differentiation of Eq. (3.2) with respect to $u$ gives

$$
\frac{\partial^{2} x}{\partial t \partial u}(t, u)=1
$$

from which

$$
\frac{\partial x}{\partial u}(t, u)=x_{0}^{\prime}(u)+t, \quad \text { for } \quad(t, u) \in\left[0, t^{*}\right) \times\left[u_{R}, u_{L}\right] .
$$

Let $t^{*}=\min _{u}\left[-x_{0}^{\prime}(u)\right]=-x_{0}^{\prime}\left(u^{*}\right)$ denote the time of shock formation; the shock location is $x^{*}=x\left(u^{*}, t^{*}\right)$. Since $x_{0}^{\prime}(u)<0$, the derivative $\partial_{u} x(t, u)<0$ as long as $t<t^{*}$.

\subsection{Solution after Shock Formation}

At times $t$ larger than $t^{*}=-x_{0}^{\prime}\left(u^{*}\right)$ (i.e., once the shock forms), Eq. (3.2) is no longer valid. In the $(x, u)$ plane, we would use the entropy (Rankine-Hugoniot) condition to construct a weak formulation of Burgers' equation. Its analog in the $(u, x)$ plane gives an equation for the shock speed $s$ :

$$
s=\frac{1}{2} \frac{u_{1}^{2}-u_{2}^{2}}{u_{1}-u_{2}}=\frac{u_{1}+u_{2}}{2},
$$

where $u_{1}(t)$ and $u_{2}(t)$ are defined as the limits of $u(t)$ from the top and bottom of the shock, respectively. They are computed as solutions of a system of ordinary differential equations (see Li et al., 2018 for detailed derivation):

$$
\left\{\begin{aligned}
\frac{\mathrm{d} u_{1}}{\mathrm{~d} t} & =\frac{1}{2} \frac{u_{1}-u_{2}}{\psi\left(u_{1}\right)-t} \\
\frac{\mathrm{d} u_{2}}{\mathrm{~d} t} & =-\frac{1}{2} \frac{u_{1}-u_{2}}{\psi\left(u_{2}\right)-t}
\end{aligned}\right.
$$

where $\psi(u) \equiv-x_{0}^{\prime}(u)$. These ODEs are subject to initial conditions $u_{1}\left(t^{*}\right)=u^{*}$ and $u_{2}\left(t^{*}\right)=$ $u^{*}$. Since $s=\mathrm{d} x^{*}(t) / \mathrm{d} t$, an equation for the shock trajectory $x^{*}(t)$ is

$$
\frac{\mathrm{d} x^{*}(t)}{\mathrm{d} t}=\frac{u_{1}+u_{2}}{2}
$$

\subsection{Summary of Hodograph Solution}

Under Assumption 3.1, the hodograph-transformed Burgers' equation [Eq. (2.13)] takes the form of the following ODEs for $x(t, u)$ :

$$
\begin{cases}t<t^{*}: & \text { Eq. (3.2) } \\ t>t^{*}: & \begin{cases}\text { Eq. (3.2) } & \text { for } u \in\left(u_{R}, u_{2}\right) \cup\left(u_{1}, u_{L}\right) \\ \text { Eq. (3.7) } & \text { for } u \in\left(u_{2}, u_{1}\right),\end{cases} \end{cases}
$$

where $t^{*}=-x_{0}^{\prime}\left(u^{*}\right)$, and $u_{1}$ and $u_{2}$ are solutions of Eq. (3.6).

Remark 3.1. We can show that $u_{1}(t)$ is a monotonically increasing function and $u_{2}(t)$ is a monotonically decreasing function, such that

$$
u_{1} \geq u^{*}, \quad u_{2} \leq u^{*}, \quad x_{0}^{\prime}\left(u_{1}\right)+t \leq 0, \quad x_{0}^{\prime}\left(u_{2}\right)+t \leq 0 .
$$


In many cases of interests, and in some our numerical experiments, either $u_{1}$ and $u_{2}$ are known or $\left|u_{2}-u_{R}\right|,\left|u_{1}-u_{L}\right| \ll \Delta t$ (so that $u_{2} \approx u_{R}$ and $u_{1} \approx u_{L}$ ). This allows one to focus on shock propagation, that is on Eq. (3.7), without having to solve Eq. (3.6).

Remark 3.2. Functions $u_{0}(x)$ that do not satisfy Assumption 3.1, such as Eq. (2.17), require a decomposition of the initial data into monotonic parts. Each monotonic piece of $u_{0}(x)$ would have a unique inverse function $x_{0}(u)$. The entropy condition implies that the increasing $x_{0}(u)$, $x_{0}^{\prime}(u)>0$, results in a rarefaction solution, which satisfies Eq. (3.2). The union of the rarefaction pieces and shock pieces would give the full solution.

Remark 3.3. The inviscid Burgers' equation is an example of hyperbolic conservation laws with monotonically increasing flux functions $F(\cdot, u)$. Generalization to hyperbolic conservation laws with a convex flux is presented in Appendix A.

\section{PHYSICS-AWARE DMD FOR CONSERVATION LAWS WITH SHOCKS}

Previous theoretic investigations, for example by Lu and Tartakovsky (2020b), demonstrated that the key to the success of the DMD in capturing nonlinear dynamics is to identify the underlying Koopman operator. Several numerical studies (Kutz et al., 2018; Rowley et al., 2009; Williams et al., 2015) confirmed this finding. The Koopman operator theory ensures that a DMD algorithm utilizes all relevant physical information to learn the dynamics. We refer to this approach as physics-aware DMD to distinguish it from the conventional DMD that learns only from (simulations-generated) data.

We review the Koopman operator theory and analyze its connection with hodograph transformation in the context of hyperbolic conservation laws. Then, we present our general framework for physics-aware DMD for problems with discontinuous solutions and shocks.

A suitable spatial discretization of Eq. (2.1) leads to a nonlinear dynamical system

$$
\frac{\mathrm{d} \mathbf{u}}{\mathrm{d} t}=\mathcal{N}(\mathbf{u})
$$

where $\mathbf{u}(t)=\left(u_{1}, \ldots, u_{J_{x}}\right)^{\top} \in \mathcal{M} \subset \mathbb{R}^{J_{x}}$ is the solution vector with $u_{j}(t)=u\left(x_{j}, t\right)$ and discretization nodes $x_{j}\left(j=1, \ldots, J_{x}\right)$; and $\mathcal{N}$ is a finite-dimensional nonlinear operator. A flow map $\mathcal{N}_{\Delta t}: \mathcal{M} \rightarrow \mathcal{M}$,

$$
\mathcal{N}_{\Delta t}\left[\mathbf{u}\left(t_{0}\right)\right] \equiv \mathbf{u}\left(t_{0}+\Delta t\right)=\mathbf{u}\left(t_{0}\right)+\int_{t_{0}}^{t_{0}+\Delta t} \mathcal{N}[\mathbf{u}(\tau)] \mathrm{d} \tau
$$

induces the corresponding discrete-time dynamical system

$$
\mathbf{u}^{n+1}=\mathcal{N}_{\Delta t}\left(\mathbf{u}^{n}\right) .
$$

Definition 4.1 (Koopman operator: Kutz et al., 2016). Consider a state $\mathbf{u}$ on a smooth $J_{x^{-}}$ dimensional manifold $\mathcal{M}$, whose dynamics are described by Eq. (4.1). The Koopman operator $\mathcal{K}$ is an infinite-dimensional linear operator that acts on all observable functions $g: \mathcal{M} \rightarrow \mathbb{C}$ such that

$$
\mathcal{K} g(\mathbf{u})=g[\mathcal{N}(\mathbf{u})] .
$$

The discrete-time Koopman operator $\mathcal{K}_{\Delta t}$ for the discrete dynamical system [Eq. (4.3)] is defined as

$$
\mathcal{K}_{\Delta t} g\left(\mathbf{u}^{n}\right)=g\left[\mathcal{N}_{\Delta t}\left(\mathbf{u}^{n}\right)\right]=g\left(\mathbf{u}^{n+1}\right) .
$$

Volume 2, Issue 1, 2021 
A major benefit of the Koopman operator is the transformation of the finite-dimensional nonlinear problem [Eq. (4.3)] in the state space into an infinite-dimensional linear problem [Eq. (4.5)] in the observable space. Since $\mathcal{K}_{\Delta t}$ is an infinite-dimensional linear operator, it has an infinite number of eigenvalues $\left\{\lambda_{k}\right\}_{k=1}^{\infty}$ and eigenfunctions $\left\{\phi_{k}\right\}_{k=1}^{\infty}$. In practice, we must make a finite approximation of the eigenvalues and eigenfunctions. The following assumption is essential to the finite approximation and the choice of observables:

Assumption 4.1. Consider a vector of $P$ observables y,

$$
\mathbf{y}^{n}=\mathbf{g}\left(\mathbf{u}^{n}\right)=\left[\begin{array}{c}
g_{1}\left(\mathbf{u}^{n}\right) \\
\vdots \\
g_{P}\left(\mathbf{u}^{n}\right)
\end{array}\right], \quad g_{p}: \mathcal{M} \rightarrow \mathbb{C} \text { is an observable function } p=1, \ldots, P .
$$

Let $\mathbf{g}$ be restricted to an invariant subspace spanned by eigenfunctions of the Koopman operator $\mathcal{K}_{\Delta t}$.

Under this assumption, $\mathbf{g}$ induces a linear operator $\mathbf{K}$ that is finite-dimensional and advances these eigen-observable functions on this subspace (Brunton et al., 2016). The physics-aware DMD Algorithm 2 can be applied to approximate the eigenvalues and eigenfunctions of $\mathbf{K}$ from snapshots data collected in the observable space.

There is no principled way to choose the observables without expert knowledge of a system under consideration. Selection of observables remains a grand challenge and an active research area; for example, machine learning and deep learning techniques were recently employed to identify the underlying Koopman operator (Morton et al., 2018). In the context of conservation laws with shocks, the equivalency between hodograph transformation and the Koopman operator, established in this study, facilitates a "smart" choice of the observables. It is implemented via the Algorithm 2.

Remark 4.1. Numerically, $\mathbf{g}$ can be obtained by interpolation from a uniform mesh in the $(x, u)$ plane to a uniform mesh in the $(u, x)$ plane, and so can $\mathbf{g}^{-1}$. The monotonicity Assumption 3.1 ensures that the observable functions are one-to-one maps.

Remark 4.2. The challenge of incorporating the shock information into the Lagrangian DMD algorithm of Lu and Tartakovsky (2020a) is the dependence of shock speed on the dependent variable $u$. Hodograph transformation facilitates the incorporation of this implicitly nonlinear information by turning $u$ into an independent variable and by rendering the shock speed given by the Rankine-Hugoniot condition linear.

Remark 4.3. For problems with shocks, we need to collect snapshots until and after a shock forms. Otherwise, the Koopman operator cannot learn the shock dynamics.

Remark 4.4. For mixed wave problems, we need to collect snapshots until and after all forms of propagation occur. This requires preobservation, preprocessing, and understanding of the data. General initial data $u_{0}(x)$ have to be separated into monotonic subregions. Physical quantities, such as shock speed and intersection point of shock and rarefaction propagation, must be understood from given data features. They give an explicit form of the shock observable function $g_{P}$; although problem-dependent, all the shock information is linear with respect to $u$. 
Algorithm 2: Physics-aware DMD algorithm

0. Create data matrices of $(M-1)$ observables, $\mathbf{Y}_{1}$ and $\mathbf{Y}_{2}$,

$$
\mathbf{Y}_{1}=\left[\begin{array}{cccc}
\mid & \mid & & \mid \\
\mathbf{y}^{1} & \mathbf{y}^{2} & \ldots & \mathbf{y}^{M-1} \\
\mid & \mid & & \mid
\end{array}\right], \quad \mathbf{Y}_{2}=\left[\begin{array}{cccc}
\mid & \mid & & \mid \\
\mathbf{y}^{2} & \mathbf{y}^{3} & \ldots & \mathbf{y}^{M} \\
\mid & \mid & & \mid
\end{array}\right]
$$

Each column of these matrices is given by

$$
\mathbf{y}^{n}=\mathbf{g}\left(\mathbf{u}^{n}\right)=\left[g_{1}\left(\mathbf{u}^{n}\right), \ldots, g_{P}\left(\mathbf{u}^{n}\right)\right]^{\top} .
$$

Here, $g_{p}^{n}=x\left(n \Delta t, \mathbf{u}^{n}\right)$ with $p=1, \ldots, P-1$ is the inverse function of $u(t, x)$, evaluated at the prescribed mesh $\tilde{\mathbf{u}}$. The last observable $g_{P}^{n}$ is a problem-dependent recording of shock information.

1. Apply SVD: $\mathbf{Y}_{1} \approx \mathbf{U} \boldsymbol{\Sigma} \mathbf{V}^{*}$, with orthogonal matrices $\mathbf{U} \in \mathbb{C}^{P \times r}$ and $\mathbf{V} \in \mathbb{C}^{(M-1) \times r}$ and diagonal matrix $\boldsymbol{\Sigma} \in \mathbb{C}^{r \times r}$, where $r$ denotes the truncated rank chosen by certain criteria, for example, Eq. (2.8).

2. Compute $\tilde{\mathbf{K}}=\mathbf{U}^{*} \mathbf{Y}_{2} \mathbf{V} \boldsymbol{\Sigma}^{-1}$ as an $r \times r$ low-rank approximation for $\mathbf{K}$.

3. Compute eigen-decomposition of $\tilde{\mathbf{K}}: \tilde{\mathbf{K}} \mathbf{W}=\mathbf{W} \boldsymbol{\Lambda}, \boldsymbol{\Lambda}=\left(\lambda_{k}\right)$.

4. Reconstruct eigen-decomposition of $\mathbf{K}$. Eigenvalues are $\boldsymbol{\Lambda}$ and eigenvectors are $\mathbf{\Phi}=\mathbf{U W}$.

5. Future $\mathbf{y}_{\mathrm{DMD}}^{n+1}$ is predicted by

$$
\mathbf{y}_{\mathrm{DMD}}^{n+1}=\mathbf{\Phi} \boldsymbol{\Lambda}^{n+1} \mathbf{b}, \quad n>M,
$$

with $\mathbf{b}=\boldsymbol{\Phi}^{-1} \mathbf{y}_{1}$.

6. Transform from observables back to state-space:

$$
\mathbf{u}_{\mathrm{DMD}}^{n}=\mathbf{g}^{-1}\left(\mathbf{y}_{\mathrm{DMD}}^{n}\right) .
$$

Remark 4.5. The error of our physics-aware DMD algorithm stems from two sources. The first is the error due to order reduction in the observable space; it represents the accuracy with which [Eq. (4.9)] predicts the true observable $\mathbf{y}^{n+1}$. According to the error estimator of Lu and Tartakovsky (2020b), this prediction accuracy depends on the number of snapshots $M$; the rank truncation criteria, as in Eq. (2.8); and the linear operator $\mathbf{K}$ induced by $\mathbf{g}$. The second is the error due to forward and backward mapping $\mathbf{g}$; in the discrete setting, it presents an error in projection between the $(x, u)$ space and the $(u, x)$ space. The model-order-reduction error and projection error are studied numerically in Section 5.6.

\section{NUMERICAL TESTS}

We apply the physics-aware DMD to construct ROMs of scalar conservation laws in different scenarios, including a shock, rarefaction, and a mixture of both. These hyperbolic conservation laws take the forms of the inviscid Burgers' equation (Sections 5.1-5.4), the Buckley-Leverett

Volume 2, Issue 1, 2021 
equation (Section 5.5), and the Euler equations (Section 5.6). The conservative first-order upwind scheme [Eq. (2.2)] is employed as a reference solution (using $J_{x}=J_{u}$ ), except when an analytical solution is available. The rank truncation criterion [Eq. (2.8)] with $\varepsilon=10^{-4}$ is used in all cases.

The observable data $\mathbf{y}^{n}$ in Eq. (4.8) rely on the mesh $\tilde{\mathbf{u}}=\left[\tilde{u}_{1}, \ldots, \tilde{u}_{J_{u}}\right]^{\top}$ with mesh size $\Delta \tilde{u}=\tilde{u}_{j+1}-\tilde{u}_{j}$ and the minimum and maximum values $\tilde{u}_{1}$ and $\tilde{u}_{J_{u}}$, respectively. In general, there are two strategies of collecting data on mesh $\tilde{\mathbf{u}}$ :

(1) Solving Eq. (3.8) for $x(t, u)$ in the $(u, x)$ plane on the mesh $\tilde{\mathbf{u}}$ at discrete time $t^{n}, n=$ $1, \ldots, M$

(2) Solving Eq. (2.2) for $u(t, x)$ in the $(x, u)$ plane on the mesh $\tilde{\mathbf{x}}$ at discrete time $t^{n}, n=$ $1, \ldots, M$, then interpolating for $x(t, u)$ in the $(u, x)$ plane on the mesh $\tilde{\mathbf{u}}$.

In the case of Burgers' equation and the Buckley-Leverett equation, both strategies are applicable. We choose strategy (1) for the examples in Sections 5.1-5.5 to avoid the projection error due to the mapping between the $(x, u)$ and $(u, x)$ planes. In the case of the Euler equations (Section 5.6), only strategy (2) is feasible. Regardless of the strategy used, the physics-aware DMD of Algorithm 2, applied to the $M$ observables $\mathbf{y}^{1}, \ldots, \mathbf{y}^{M}$ in Eq. (4.8), allows us to predict $\mathbf{y}_{\mathrm{DMD}}^{n+1}$ for $n>M$ and to obtain $\mathbf{u}_{\mathrm{DMD}}^{n}$ by concatenating the interpolation of $\mathbf{y}_{\mathrm{DMD}}^{n+1}$ on the mesh for $x$.

\subsection{Riemann Problem for Burgers' Equation with Shock}

Consider the inviscid Burgers' equation [Eq. (2.13)] defined for $(x, t) \in[-0.5,1.5] \times[0,1]$ and with initial data

$$
u_{0}(x)= \begin{cases}2 & \text { for }-0.5 \leq x<0 \\ 0 & \text { for } 0 \leq x \leq 1.5\end{cases}
$$

This problem admits an analytical solution

$$
u(x, t)= \begin{cases}2 & \text { for } \quad-0.5 \leq x<s t \\ 0 & \text { for } \quad s t<x \leq 1.5,\end{cases}
$$

where the shock speed $s=1$ is determined from the Rankine-Hugoniot condition.

The discontinuous initial data $u_{0}(x)$ in Eq. (5.2) do not satisfy Assumption 3.1. Thus we approximate the step function $u_{0}(x)$ with a smooth function; for example the hyperbolic tangent

$$
u_{0} \approx 1-\tanh \left(\frac{x}{\delta}\right), \quad \delta \ll 1,
$$

which satisfies Assumption 3.1. In the $(u, x)$ plane,

$$
x_{0} \approx \frac{\delta}{2} \log \left(\frac{2-u_{0}}{u_{0}}\right), \quad \delta \ll 1 .
$$

This approximation is valid in the neighborhood of the shock interface; away from it, Eq. (5.1) is used. It follows from Eq. (5.4) that

$$
\text { - } x_{0}^{\prime}(u)=\delta /[(u-2) u]<0 \text { for } u \in(0,2) \text {. }
$$


- $x_{0}(u)$ has a unique inflection point at $\left(u^{*}=1, x^{*}=0\right)$.

- $x_{0}^{\prime \prime \prime}(u)=\left[4\left(3 u^{2}-6 u+4\right)\right] /\left[(u-2)^{3} u^{3}\right]$ and thus $x_{0}^{\prime \prime \prime}\left(u^{*}\right)=-4<0$.

Snapshots of $x(t, u)$ on a uniform mesh $\tilde{\mathbf{u}}=\left[\tilde{u}_{1}, \ldots, \tilde{u}_{J_{u}}\right]^{\top}$, which consists of $J_{u}=2000$ equidistant points, are collected at $M=250$ times until $T=0.25$. The ROM is used to predict the solution $u(x, t)$ for larger times, $t>T$. The shock-related information is contained in $u_{1}(t)$ and $u_{2}(t)$, first defined in Eq. (3.5). Since both are constant in this example, no extra observable $g_{P}$ is needed to record the shock information. Each column $\mathbf{y}^{n}$ in the data matrix is of length $P=2000$ and elements $\mathbf{y}_{j}^{n}=x\left(t^{n}, \tilde{u}_{j}\right)$ for $j=1, \ldots, J_{u}$. If we were to add the constant shock information $u_{1}$ and $u_{2}$ to the observables, then $\mathbf{y}^{n}$ would have length $P=2002$ such that elements $\mathbf{y}_{j}^{n}=x\left(t^{n}, \tilde{u}_{j}\right)$ for $j=1, \ldots, J_{u}$ are supplemented with two extra elements $\mathbf{y}_{2001}^{n}=u_{1}=2$ and $\mathbf{y}_{2002}^{n}=u_{2}=0$. Doing so would lead to the same prediction: the DMD algorithm is able to learn the pattern of the last two observables being constant in time.

Figure 3 demonstrates that the physics-aware DMD algorithm with hodograph transformation captures the behavior of the shock propagation. Only $r=2$ modes are needed to construct the ROM, which remains accurate for a relatively long time in the extrapolation mode. Hodograph transformation converts the nonlinear conservation law [Eq. (2.13)] with discontinuous initial data [Eq. (5.1)] into a linear shift with constant speed, which is readily learned from data. Due to the monotonicity constraint, the solution using hodograph transformation (and the concomitant DMD prediction) is only valid in the neighborhood of the shock interface, which is often of interest in itself. Away from the discontinuity, the Lagrangian DMD (Lu and Tartakovsky, 2020a) is accurate and should be used instead.

\subsection{Riemann Problem for Burgers' Equation with Rarefaction Wave}

Consider the inviscid Burgers' equation [Eq. (2.13)] defined for $(x, t) \in[-1,1] \times[0,1]$ and with initial data

$$
u_{0}(x)= \begin{cases}-1 & \text { for }-1 \leq x<0 \\ 1 & \text { for } 0 \leq x \leq 1\end{cases}
$$

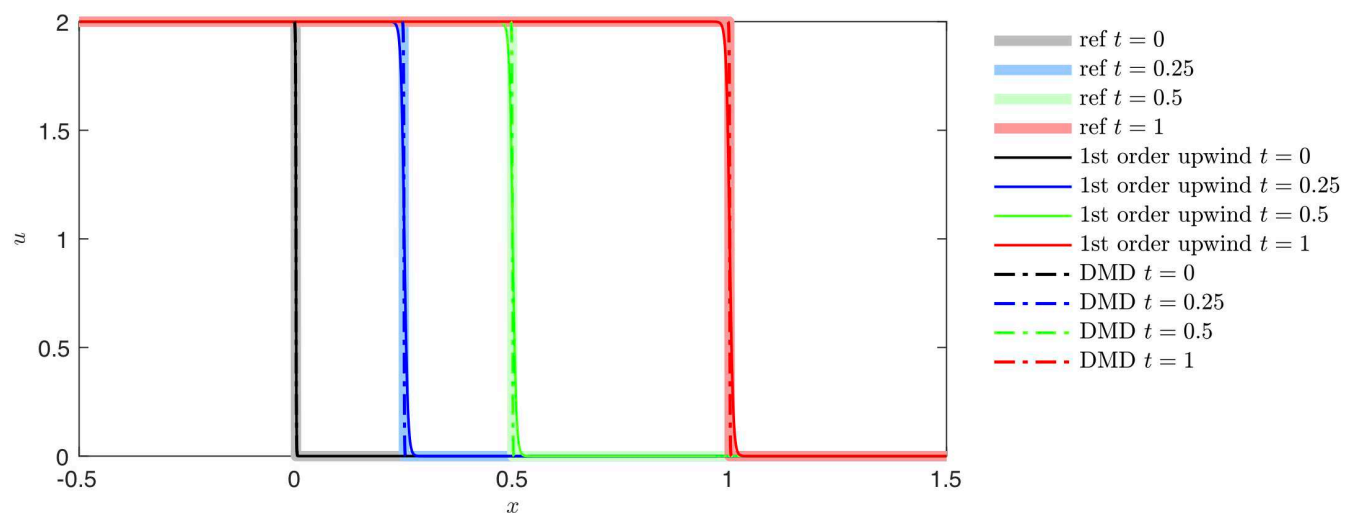

FIG. 3: Physics-aware DMD solution of the inviscid Burgers' equation with a shock. The reference solution is given by analytic solution (5.2). 1st order upwind scheme by Eq. (2.2) is also plotted here in solid line.

Volume 2, Issue 1, 2021 
This problem admits an analytical solution in the form of a rarefaction wave,

$$
u(x, t)= \begin{cases}-1 & \text { for } \quad-1 \leq x<-t \\ x / t & \text { for } \quad-t<x<t \\ 1 & \text { for } t<x \leq 1\end{cases}
$$

A hyperbolic-tangent approximation analogous to Eq. (5.3) is used to deal with the discontinuity in the initial data $u_{0}(x)$. And the same structure of data matrix is used in the physics-aware DMD algorithm 2 with $J_{u}=2000$ and $M=250$ until $t=0.25$. Since there is no shock in this scenario, no extra observable is needed to record shock-related information.

Figure 4 shows the same satisfactory ROM results for this problem with a rarefaction wave. Only $r=2$ modes are needed to obtain accurate predictions because the hodograph transform, $x=x(t, u)$, satisfies a linear ODE [Eq. (3.2)], with $u$ acting as an independent variable. The absence of a shock suggests that this rarefaction scenario can also be handled with the Lagrangian DMD algorithm, with similar results.

Given an accurate approximation of the initial discontinuity (i.e., selecting $\delta$ to be sufficiently small) the ROM trained on the data generated from the solution of Eq. (3.2) is even more accurate than the HFM solution. The upwind scheme [Eq. (2.2)] has first-order accuracy, $O(\Delta t)$, while the physics-aware DMD algorithm can have spectral accuracy. Figures 3 and 4 show that the physics-aware DMD solution has a much sharper interface than that estimated with the firstorder upwind scheme [Eq. (2.2)].

\subsection{Smooth Solution of Riemann Problem for Burgers' Equation with Nonmonotonic Initial Data}

Consider Burgers' equation [Eq. (2.13)] defined for $(x, t) \in[-\pi / 2,3 \pi / 2] \times[0,1]$ and with initial data

$$
u_{0}(x)=1+\sin (x)
$$

Since these initial data violate Assumption 3.1, we decompose the interval $[0,2 \pi]$ into two parts: in the left part, $x \in[-\pi / 2, \pi / 2], u_{0}(x)$ monotonically increases; in the right part, $x \in$

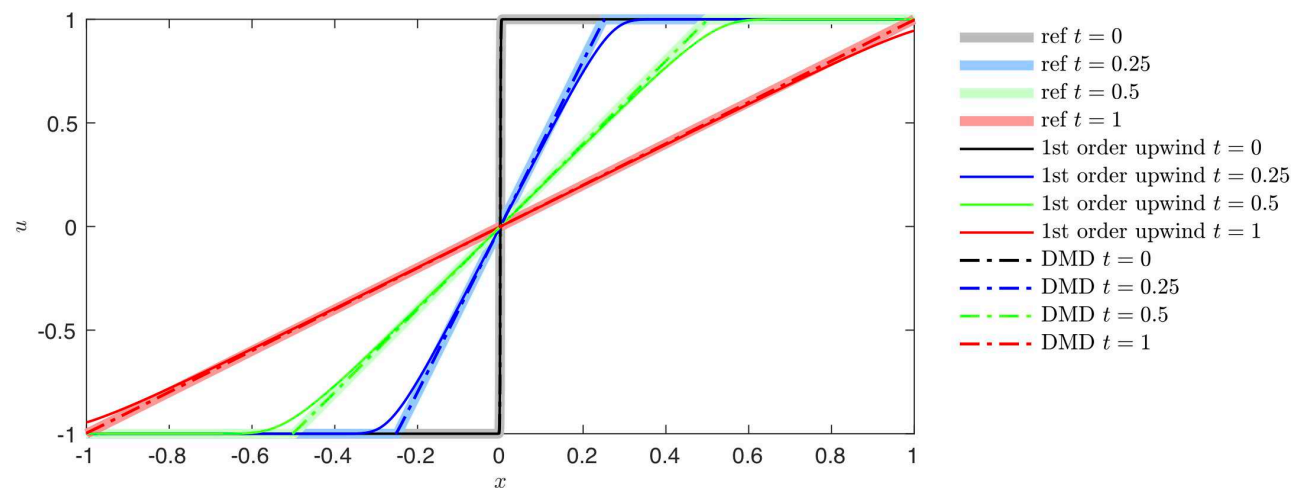

FIG. 4: Physics-aware DMD solution of the inviscid Burgers' equation with a rarefaction wave. The reference solution is given by analytic solution [Eq. (5.6)]. First order upwind scheme by Eq. (2.2) is also plotted here in solid line. 
$[\pi / 2,3 \pi / 2]$, it monotonically decreases. Each part has a unique inverse function of $x_{0}\left(t, u_{0}\right)$; we denote $x_{l}(t, u)$ as the evolution of the left part and $x_{r}(t, u)$ as the evolution of the right part. Since the shock formation time is $t^{*}=1$, the equation of characteristics for this Riemann problem is equivalent to Eq. (3.2) on any finite-time interval $[0, T] \subset[0,1]$. Although this is a shock-free scenario, the two parts have different wave propagation behaviors. The numerical scheme (2.2) with $J_{x}=2000$ spatial discretization points and $N=1000$ time steps provides the reference solution. The data used to inform our DMD method consist of $M=250$ snapshots with observables $\mathbf{y}_{p}^{n}=x_{l}\left(t^{n}, \tilde{u}_{p}\right)$ for $p=1, \ldots, J_{u}$ and $\mathbf{y}_{p}^{n}=x_{r}\left(t^{n}, \tilde{u}_{p-J_{u}}\right)$ for $p=J_{u}+1, \ldots, 2 J_{u}$. No extra observable is needed to record shock-related information since there is no shock formation in the considered time interval.

Figure 5 demonstrates the ability of the ROM based on our physics-aware DMD algorithm to capture these nonlinear dynamics. Only $r=2$ modes are needed to obtain accurate predictions due to the linearity after hodograph transform. The ROM was trained on the early $(t \leq 0.25)$ data, which exhibit smooth gradients. Yet, it accurately captures sharp gradients at later times (e.g., $t=1)$. That is because, in the $(u, x)$ domain of the hodograph transform, higher gradients of $u(\cdot, x)$ translate into flatter horizontal plots of $x(\cdot, u)$.

\subsection{Riemann Problem for Burgers' Equation with Rarefaction and Shock}

Consider Burgers' equation [Eq. (2.13)] defined for $(x, t) \in[0,2] \times[0,1]$ and with the Gaussiantype initial data in Eq. (2.17). This is the setting we used to illustrate the failure of the Lagrangian DMD in Section 2.3 (Fig. 2). The numerical scheme [Eq. (2.2)] with $J_{x}=2000$ spatial discretization points and $N=10^{5}$ time steps provides the reference solution. The finer time discretization is needed to satisfy the CFL constraints. The data used to inform our physics-aware DMD method consist of $M=3000$ snapshots of solving Eq. (3.8). These data are sufficiently rich to identify the rarefaction and shock behavior of the solution.

A decomposition of the initial data $u_{0}(x)$ in Eq. (2.17) is needed to enforce monotonicity. The increasing branch of $u_{0}(x)$ is responsible for the rarefaction and its decreasing branch gives rise to the shock. It follows from Eq. (2.17) that

$$
x_{l}\left(u, t^{0}\right)=0.3-\sqrt{-0.001 \ln (2 u-1.6)} \quad \text { and } \quad x_{r}\left(u, t^{0}\right)=0.3+\sqrt{-0.001 \ln (2 u-1.6)} .
$$

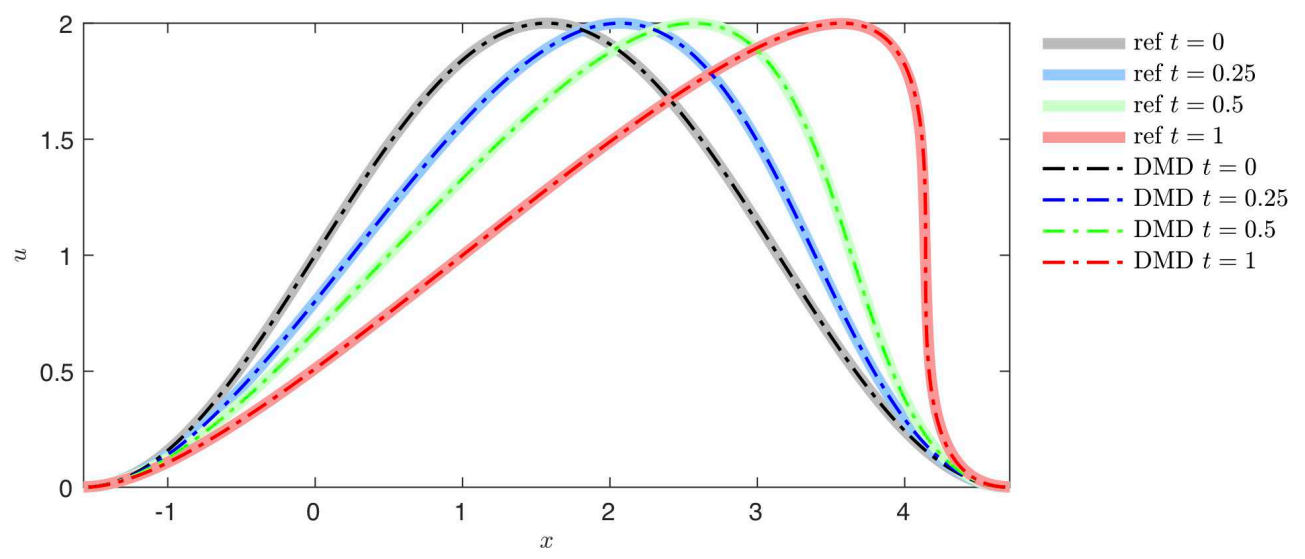

FIG. 5: Physics-aware DMD solution of the inviscid Burgers' equation subject to nonmonotonic initial condition. The reference solution is computed with Eq. (2.2).

Volume 2, Issue 1, 2021 
We can verify that $x_{r}\left(u ; t^{0}\right)$ has a unique inflection point $\left(u^{*}, x^{*}\right)$ with $u^{*}=0.8+1 /(2 \sqrt{\mathrm{e}})$ and $x^{*}=\sqrt{0.002 \mathrm{e}}$ and, for $u \in(0.8,1.3)$, both $x_{r}^{\prime}\left(u ; t^{0}\right)<0$ and $x_{r}^{\prime \prime \prime}\left(u ; t^{0}\right)<0$. Consequently, the data are generated from Eq. (3.8) as follows.

1. A uniform mesh $\tilde{\mathbf{u}}=\left[\tilde{u}_{1}, \ldots, \tilde{u}_{J_{u}}\right]^{\top}$ is constructed with mesh size $\Delta \tilde{u}=\tilde{u}_{j+1}-\tilde{u}_{j}$, and $\tilde{u}_{1}=0.8$ and $\tilde{u}_{J_{u}}=1.3$.

2. For $x_{l}(u, t)$, no shock develops. The full discretization [Eq. (3.2)] gives

$$
\left\{\begin{array}{l}
\frac{x_{l}\left(\tilde{u}_{j}, t^{n+1}\right)-x_{l}\left(\tilde{u}_{j}, t^{n}\right)}{\Delta t}=\tilde{u}_{j}, \\
x_{l}\left(\tilde{u}_{j}, t^{0}\right)=0.3-\sqrt{-0.001 \ln \left(2 \tilde{u}_{j}-1.6\right)} .
\end{array}\right.
$$

3. For $x_{r}(u, t)$, the shock formation time $t^{*}$ is calculated as

$$
t^{*}=\min _{u}\left\{-x_{r}^{\prime}\left(u, t^{0}\right)\right\} \approx 0.074 .
$$

a. If $t^{n+1} \leq t^{*}$, then

$$
\left\{\begin{array}{l}
\frac{x_{r}\left(\tilde{u}_{j}, t^{n+1}\right)-x_{r}\left(\tilde{u}_{j}, t^{n}\right)}{\Delta t}=\tilde{u}_{j} \\
x_{r}\left(\tilde{u}_{j}, t^{0}\right)=0.3+\sqrt{-0.001 \ln \left(2 \tilde{u}_{j}-1.6\right)} .
\end{array}\right.
$$

b. If $t^{n+1}>t^{*}, u_{2}\left(t^{n}\right)=u_{R}=0.8$ is known and $u_{1}\left(t^{n}\right)$ is approximated by the intersection of $x_{l}\left(u, t^{n}\right)$ and $x_{r}\left(u, t^{n}\right)$ due to the continuity of the solution; that is

$$
\left\{\begin{array}{l}
u_{1}\left(t^{n}\right)=\underset{\tilde{u}_{j}}{\operatorname{argmin}}\left|x_{r}\left(\tilde{u}_{j}, t^{n}\right)-x_{l}\left(\tilde{u}_{j}, t^{n}\right)\right|, \quad u_{2}\left(t^{n}\right)=u_{R}=0.8, \\
\frac{x_{r}\left(\tilde{u}_{j}, t^{n+1}\right)-x_{r}\left(\tilde{u}_{j}, t^{n}\right)}{\Delta t}=\frac{u_{1}\left(t^{n}\right)+u_{2}\left(t^{n}\right)}{2} .
\end{array}\right.
$$

4. The observable vector $\mathbf{y}^{n}$ of length $P=2 J_{u}+2$ comprises $\mathbf{y}_{p}^{n}=x_{l}\left(\tilde{u}_{p}, t^{n}\right)$ for $p=$ $1, \ldots, J_{u} ; \mathbf{y}_{p}^{n}=x_{r}\left(\tilde{u}_{p-J_{u}}, t^{n}\right)$ for $p=J+1, \ldots, 2 J_{u}$; and $\mathbf{y}_{2 J_{u}+1}^{n}=u_{1}\left(t^{n}\right)$ and $\mathbf{y}_{2 J_{u}+2}^{n}=u_{2}\left(t^{n}\right)$.

Figure 6 shows that the physics-aware DMD based on hodograph transformation provides an accurate ROM for this Riemann problem, which could not be treated with the original Lagrangian DMD. The physical shock information, which is needed for the last two observable functions, includes the shock speed and the intersection point of the rarefaction wave and the shock trajectory. In this setting, the shock speed varies with time but is still linear with respect to $u$. The physics-aware DMD algorithm can learn this linear relationship from the data with no difficulties. Only $r=4$ modes are needed and all of the advantages of linearity are achieved with the hodograph transform.

\subsection{Riemann Problem for Buckley-Leverett's Equation}

Consider the hyperbolic conservation law [Eq. (2.1)] with a nonmonotonic flux function,

$$
F=\frac{u^{2}}{u^{2}+a(1-u)^{2}}, \quad a=0.5,
$$




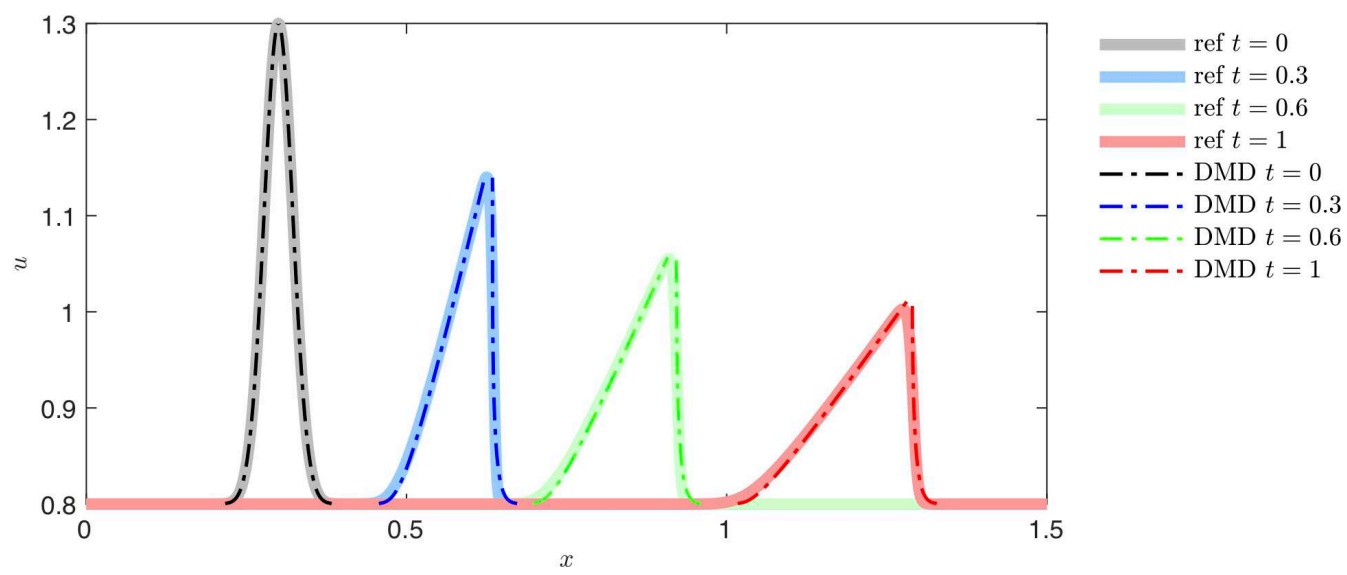

FIG. 6: Physics-aware DMD solution of the inviscid Burgers' equation with a rarefaction wave and shock. The reference solution is computed with Eq. (2.2).

that is defined for $(x, t) \in[0,2] \times[0,0.5]$ and is equipped with initial data

$$
u_{0}(x)= \begin{cases}1 & \text { for } 0 \leq x<1 \\ 0 & \text { for } 1 \leq x \leq 2\end{cases}
$$

The Buckley-Leverett equation [Eqs. (2.1) and (5.12)] with initial condition [Eq. (5.13)] is widely used to describe the injection process of immiscible two-phase flow in porous media.

Similar to Eq. (5.3), the initial discontinuity is approximated with the hyperbolic tangent function. The hodograph treatment of this more general problem is provided in Appendix A. The numerical scheme [Eq. (2.2)] with $J_{x}=2000$ spatial discretization points and $N=1000$ time steps provides the reference solution up to $t=0.5$. The data used to inform our physicsaware DMD method consist of $M=250$ snapshots of solving Eq. (A.11) with $J_{u}=2000$ discretization points until $t=0.125$. This set of snapshots is sufficiently rich to reveal a selfsimilar structure of the solution.

Although the initial data $u_{0}$ are monotonic, their decomposition is needed according to the convex hull construction of the flux function (Appendix A). The reformulation involves two branches of different linear equations with two sets of the disjoint initial data. Similar to the previous section, the last two observation functions comprises the shock speed as well as the intersection point of the rarefaction wave and the shock trajectory. This intersection point defines the magnitude of the shock and informs the convex hull construction of the flux function.

Figure 7 demonstrates that the physics-aware DMD with $r=4$ modes accurately captures the future states in a relatively long time. Hodograph transformation allows us to determine the underlying linear Koopman operator in the nonlinear conservation laws. The iteration-free feature of DMD enhances its effectiveness and efficiency.

Remark 5.1. In laboratory experiments with multiphase flows in porous media, we often measure the flow rates of two fluids and approximates the displacement profiles. Such observational data can be used to construct an effective ROM after interpolating them from the $(x, u)$ plane to the $(u, x)$ plane. Such a construction would not require any knowledge of the empirical flux function, such as Eq. (5.12), and its parameters, such as $a$.

Volume 2, Issue 1, 2021 


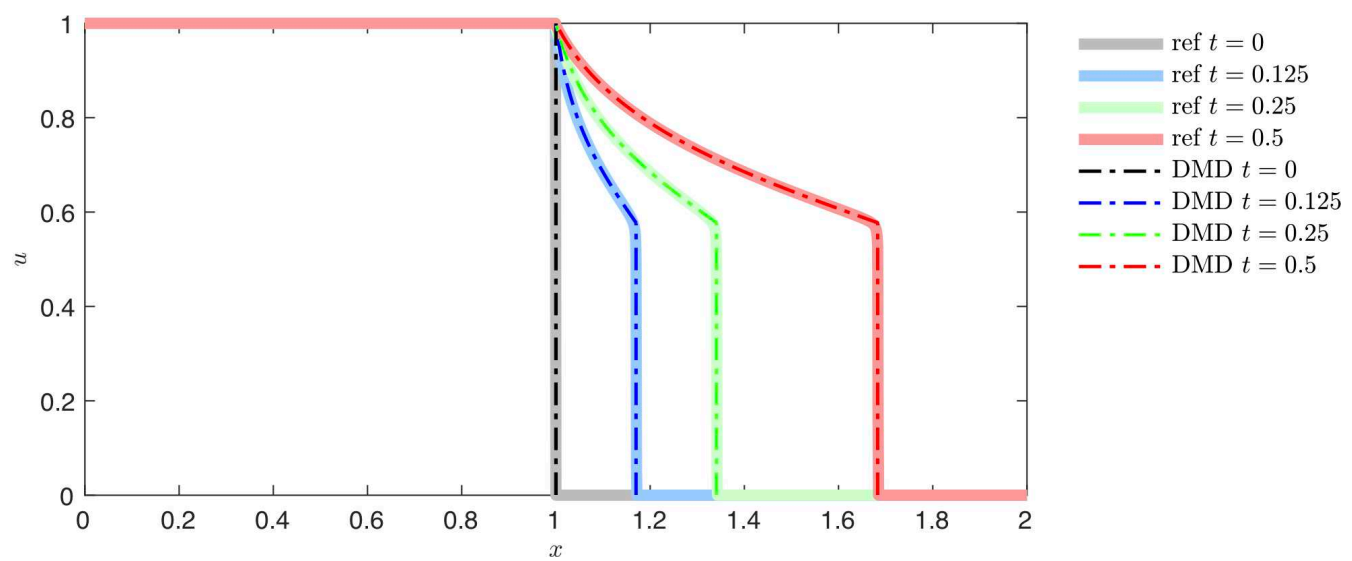

FIG. 7: Physics-aware DMD solution of the Buckley-Leverett equation, which has a nonmonotonic flux function. The reference solution is computed with Eq. (2.2).

\subsection{Riemann Problem for Euler Equations}

Consider a one-dimensional Sod shock tube problem,

$$
\begin{aligned}
& \frac{\partial}{\partial t}\left(\begin{array}{c}
\rho \\
\rho u \\
E
\end{array}\right)+\frac{\partial}{\partial x}\left(\begin{array}{c}
\rho u \\
\rho u^{2}+p \\
u(E+p)
\end{array}\right)=0, \quad(x, t) \in[-0.5,0.5] \times[0,0.25] \\
& E=\frac{p}{\gamma-1}+\frac{1}{2} \rho u^{2}, \quad \gamma=1.4
\end{aligned}
$$

with initial conditions

$$
\rho(x, 0)=\left\{\begin{array}{ll}
1 & x<0.5 \\
0.125 & x \geq 0.5
\end{array}, \quad p(x, 0)=\left\{\begin{array}{ll}
1 & x<0.5 \\
0.1 & x \geq 0.5
\end{array}, \quad u(x, 0)=0 .\right.\right.
$$

This problem admits an analytic solution obtained, for example via the method of characteristics. Figure 8 shows that the solution is a mixture of a rarefaction wave and a shock, which undermines the performance of conventional ROM approaches. For example, Fig. 9(a) shows the failure of the standard (Eulerian) DMD to construct a ROM in the $(x, \rho)$ plane, with similarly unsatisfactory performance for $p$ and $u$. In these simulations, $x$ is discretized using $J_{x}=1000$ equidistant points, and $M=250$ snapshots data are collected from $t=0$ to $t=0.0625$. The prediction at later time, $t=0.125$, already shows tremendous errors and loss of correct rarefaction/shock features.

Our hodograph-based physics-aware DMD resolves this challenge. Figure 9(b) demonstrates that it yields an accurate ROM by interpolating the $M=250$ snapshots of the analytical solution onto the $(\rho, x)$ plane in which the $\rho$ coordinate is discretized with a $J_{\rho}=1000$ equidistant mesh. Here, $x(\rho, t)$ is selected as the observable and the linear Koopman operator on $x(\rho, t)$ is efficiently represented by $r=210$ modes in the physics-aware DMD algorithm. Nearly 4/5 rank reduction is obtained and the computational time for DMD prediction is negligible due to its iteration-free nature. Although an equation for $x(\rho, t)$ is not available, the hodograph transform for one-dimensional scalar hyperbolic equations motivates this proper selection of an observable. 
The hodograph transform for multidimensional PDEs is more complicated (e.g., Li and Zheng, 2009; Mohyuddin et al., 2008) and not directly applicable to our current ROM framework. Our results demonstrate that while the connection between the hodograph transform and the Koopman theory is unclear in multiple dimensions, it can still guide the selection of observables.

Finally, we investigate the model-order reduction error $e_{x}$ and the projection error $e_{\text {pr }}$ discussed in Remark 4.5. In this example, $e_{x}$ is the DMD prediction error in terms of $x(\rho, t)$,

$$
\begin{gathered}
e_{x}^{n}=\left\|\mathbf{y}^{n}-\mathbf{y}_{\mathrm{DMD}}^{n}\right\|, \\
\mathbf{y}^{n}=\left[x\left(\rho_{1}, t^{n}\right), \cdots, x\left(\rho_{J_{\rho}}, t^{n}\right)\right]^{\top}=\mathbf{g}\left(\left[\rho\left(x_{1}, t^{n}\right), \cdots, \rho\left(x_{J_{x}}, t^{n}\right)\right]^{\top}\right) .
\end{gathered}
$$

This quantity provides a measure of the discrepancy between the reference observable values and their DMD prediction, without isolating various sources of the error. We also consider $e_{\mathrm{pr}}$, the error in creating the observable $x(\rho, t)$ via projection from the $(x, \rho)$ plane onto the $(\rho, x)$ plane during preprocessing and in estimating the solution $\rho(x, t)$ backward during post processing.
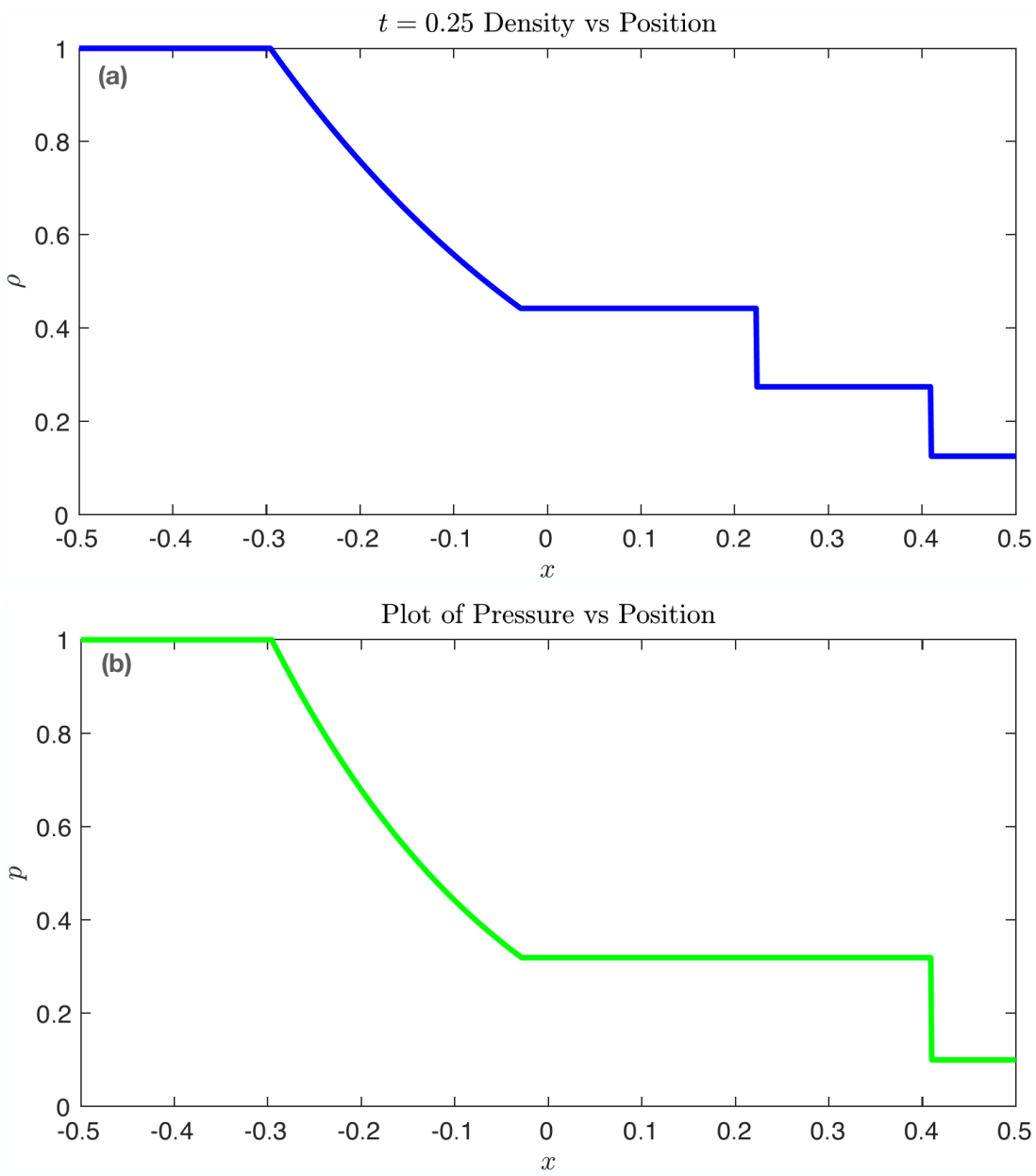

FIG. 8.

Volume 2, Issue 1, 2021 

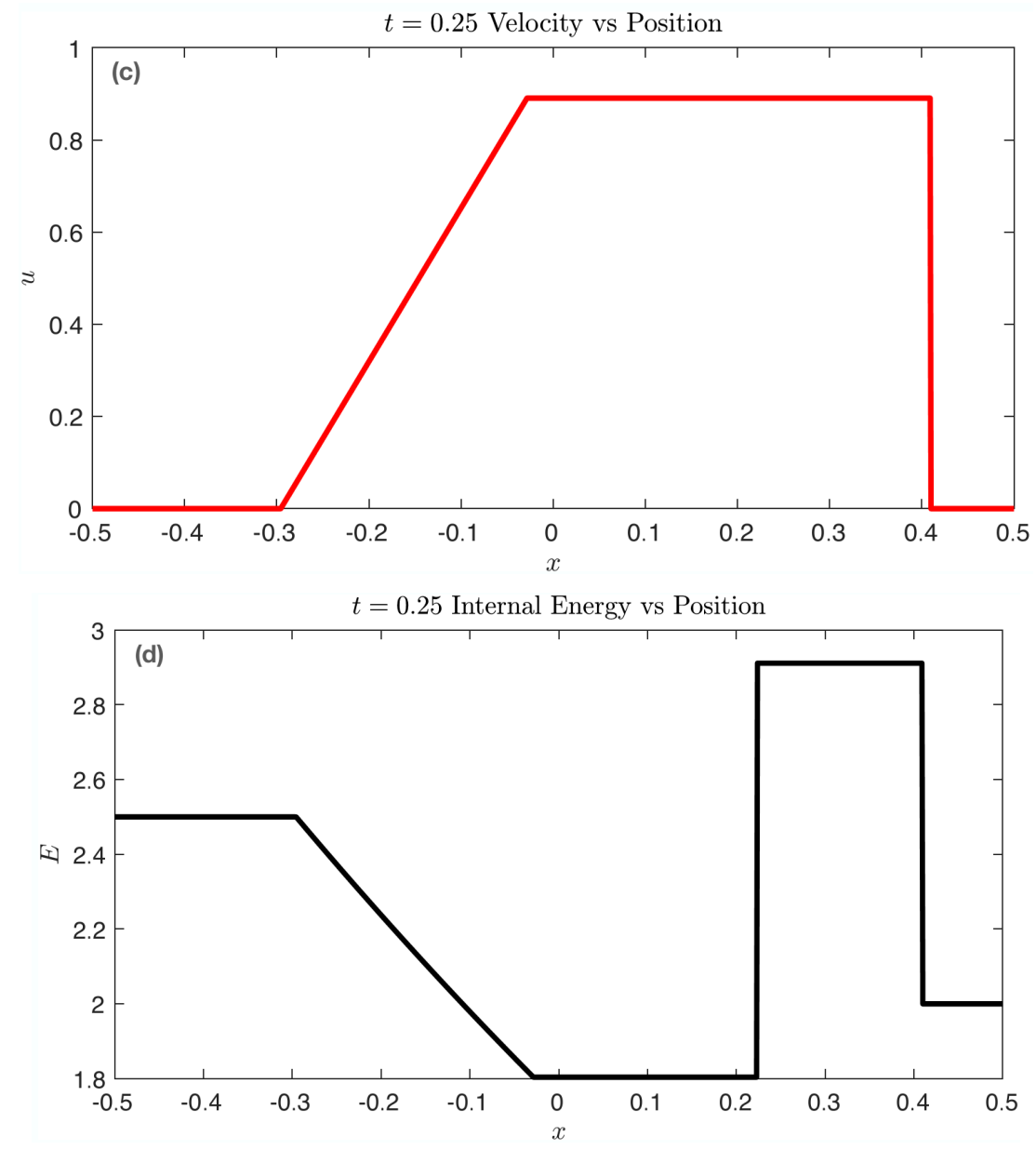

FIG. 8: The analytical solution to the Sod shock tube problem [Eq. (5.14)]: (a) density, (b) pressure, (c) velocity, and (d) energy; all evaluated at time $t=0.25$

Both $e_{x}$ and $e_{\mathrm{pr}}$ contribute to the total error $e_{\rho}$ in DMD estimation of the solution $\rho(x, t)$,

$$
e_{\rho}^{n}=\left\|\boldsymbol{\rho}^{n}-\boldsymbol{\rho}_{\mathrm{DMD}}^{n}\right\|, \quad \boldsymbol{\rho}^{n}=\left[\rho\left(x_{1}, t^{n}\right), \cdots, \rho\left(x_{J_{x}} \cdot t^{n}\right)\right]^{\top}, \quad \boldsymbol{\rho}_{\mathrm{DMD}}^{n}=\mathbf{g}^{-1}\left(\mathbf{y}_{\mathrm{DMD}}^{n}\right) .
$$

Temporal evolution of the errors $e_{x}$ and $e_{\rho}$ is plotted in Fig. 10 with both errors [Eqs. (5.16) and (5.17)] reported in $L_{2}$ norm and defined on the corresponding meshes $J_{\rho}=1000$ and $J_{x}=1000$. The model-order-reduction error $e_{x}$ decreases with the number of snapshots $M$, resulting in a more accurate prediction. This is consistent with the intuition that DMD can better capture the dynamics by learning from richer/larger data sets. For example, insufficient data ( $M=125$ ) fail to sample the essential features in the dynamics of $x(\rho, t)$. The rank truncation $\varepsilon$ also plays a crucial role in the model-order-reduction error $e_{x}$ : significant accuracy is sacrificed if essential singular values are truncated in SVD (e.g., by setting $\varepsilon=10^{-2}$ ) but retaining too many singular values, e.g., by setting $\varepsilon=10^{-4}$, increases the impact of noise. This issue has been discussed in several DMD studies (e.g., by Kutz et al., 2016). The choice of a rank-truncation criteria is nontrivial and, thus, the optimal truncation is problem-dependent. The total DMD error 

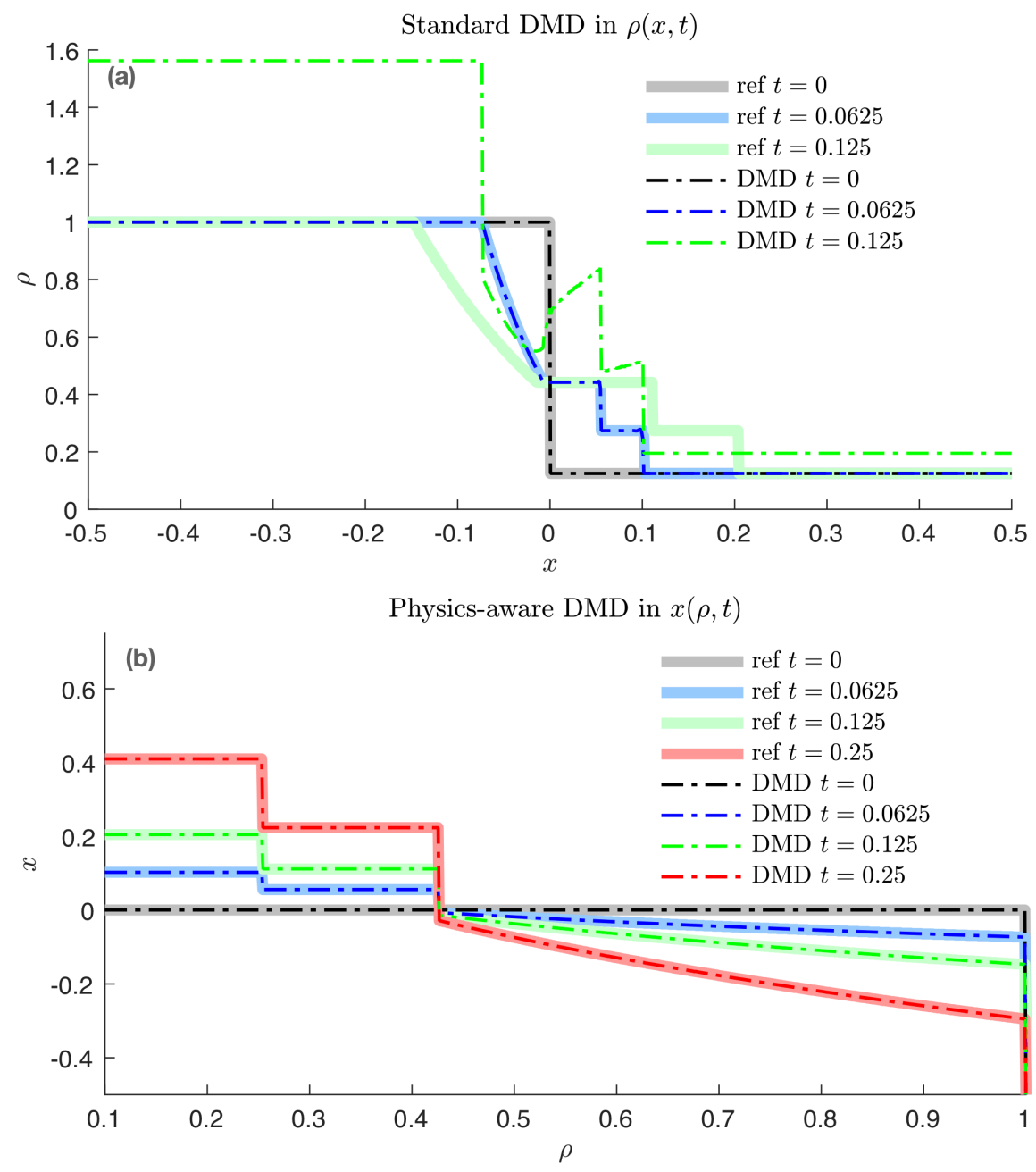

FIG. 9: ROMs constructed by (a) the standard DMD and (b) the physics-aware DMD. The former uses snapshots of $\rho(x, t)$ in the $(x, \rho)$ plane, while the latter relies on snapshots of $x(\rho, t)$ in the $(\rho, x)$ plane.

$e_{\rho}$ is shown in the bottom row of Fig. 10. In addition to $e_{x}$, this error also accounts for the projection between the $(x, \rho)$ plane and the $(\rho, x)$ plane, which was conducted via a shape-preserving interpolation method. For a well-controlled model-reduction error, $e_{x} \sim \mathcal{O}\left(10^{-2}\right)$, the total error, $e_{\rho} \sim \mathcal{O}\left(10^{-1}\right)$, is dominated by the projection error. For insufficient data $(M=125)$ or low rank truncation $\left(\varepsilon=10^{-2}\right)$, the large total error in the prediction regime, $e_{\rho} \sim \mathcal{O}(1)$, is dominated by the model-reduction error, $e_{x} \sim \mathcal{O}(1)$. The time evolution of the errors is oscillatory due to the interpolation during the transformation between observables and state-space.

\section{SUMMARY AND CONCLUSIONS}

The Lagrangian DMD (Lu and Tartakovsky, 2020a) provides a robust tool to construct ROMs of hyperbolic conservation laws, a class of problems for which standard (Eulerian) DMD methods

Volume 2, Issue 1, 2021 

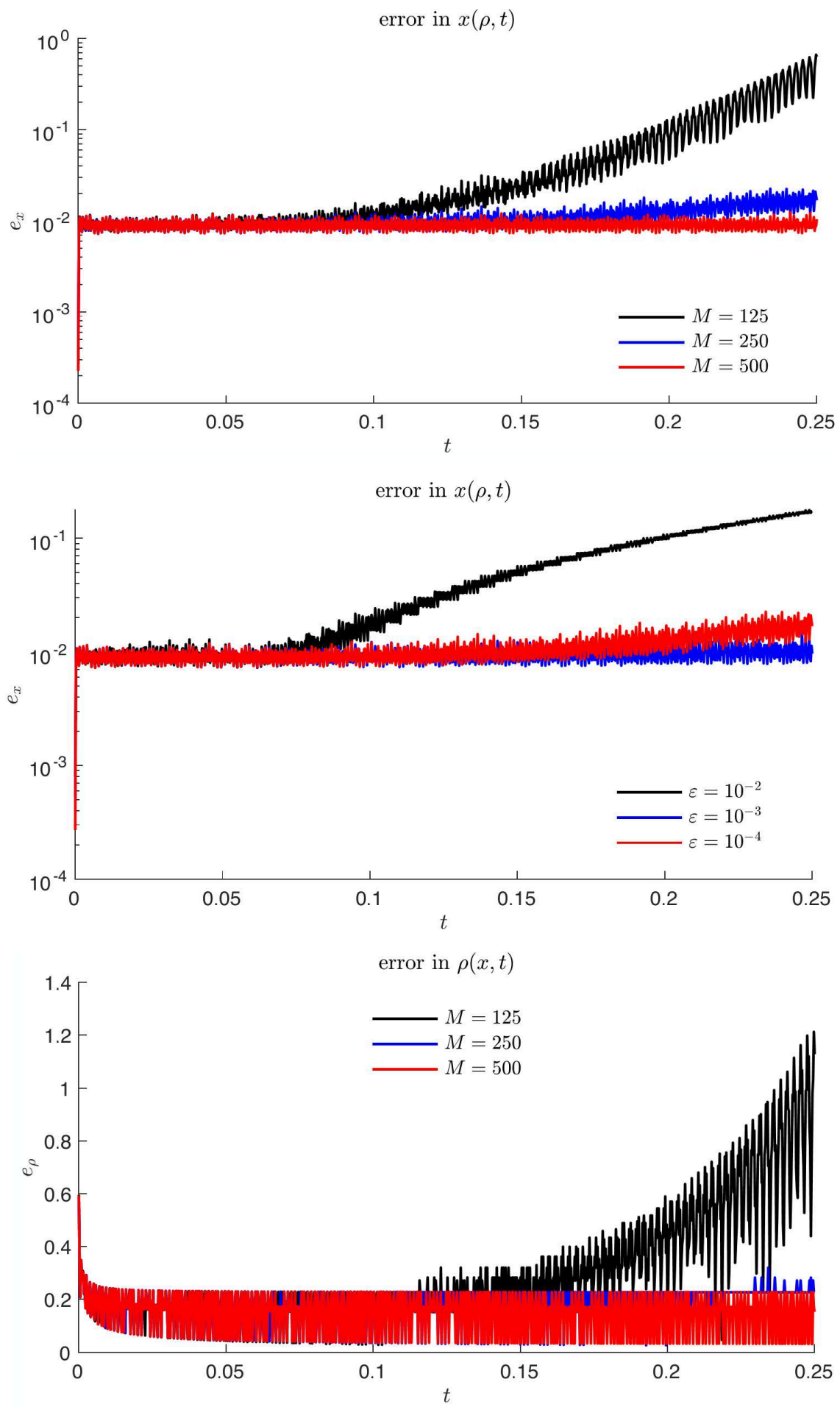

FIG. 10. 


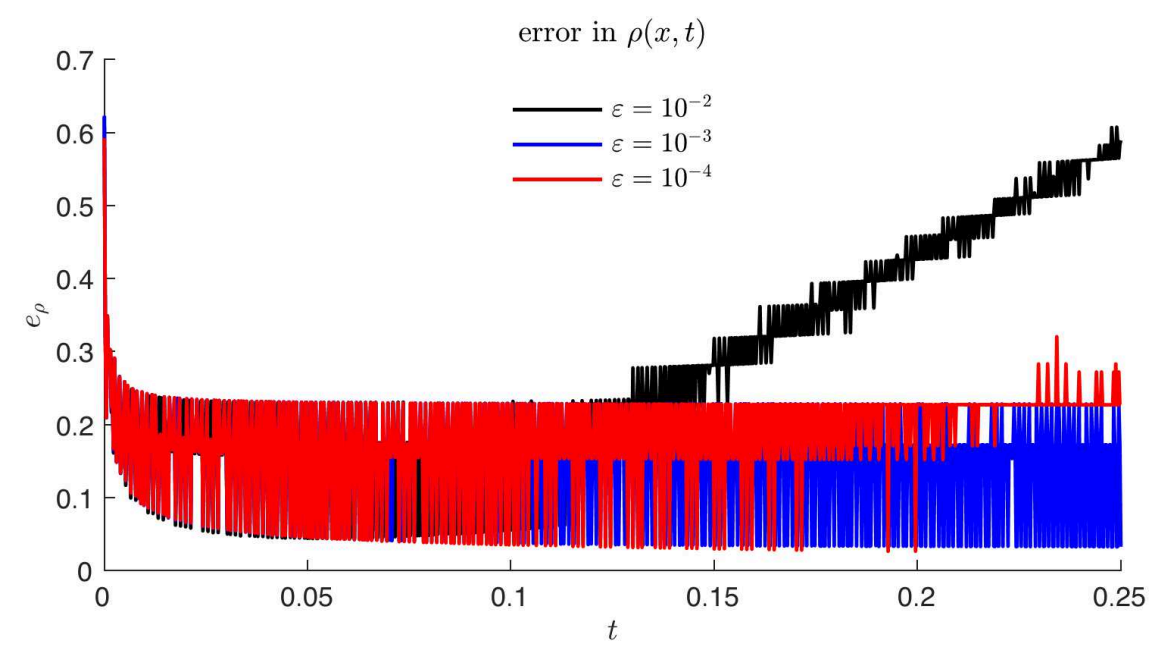

FIG. 10: Prediction errors of the ROMs constructed by the physics-aware DMD with different parameters. The top row shows the model-order reduction error $e_{x}$ defined in Eq. (5.16); the total DMD error $e_{\rho}$, defined in Eq. (5.17), is shown in the bottom row.

fail. However, this algorithm is limited to problems that admit smooth strong solutions. We extended it to problems with shocks and rarefaction waves, thus addressing a long-standing challenge in ROM construction. This challenge stems from severe grid distortion typical of Lagrangian POD and DMD algorithms. Lacking information about shocks and discontinuities, DMD mode projection from the HFM to a ROM does not preserve the topological structure of the interface where characteristic lines cross each other. We resolved this issue by combining hodograph transformation with physics-aware DMD algorithm (Lu and Tartakovsky, 2020a). The relevant research codes are available at https://github.com/DDMS-ERE-Stanford/ dmd_hodograph.

Hodograph transforms are consistent with the Koopman operator theory in that both aim to identify linear structures in the underlying nonlinear dynamics. Our physics-aware DMD algorithm enhanced by hodograph transformation is capable of predicting the dynamics of weak solutions, which satisfies the entropy condition. We demonstrated the accuracy and robustness of our algorithm on several numerical tests.

To the best of our knowledge, our study is the first to establish a connection between hodograph transformation and the Koopman operators. By providing a principled way for identifying the observables needed by the Koopman operator theory, this connection opens a door to construct ROMs for a wide range of nonlinear PDEs that are linearizable by hodograph transformation (Clarkson et al., 1989). There is an algorithmic method to do the linearization via extended hodograph transforms. As a result, we can take advantage of the linearity and design robust iteration-free physics-aware DMD. Moreover, data-driven modeling and uncertainty quantification can be further explored using this framework. Our numerical experiments demonstrated that many physical quantities, such as the shock speed in Burgers' equation and the mobility constant in the Buckley-Leverett equation, can be learned from (simulation) data as long as we analyze them in a "smart" way.

We established a connection between the hodograph transforms and the Koopman operator theory for one-dimensional scalar hyperbolic PDEs. A similar idea was carried over to

Volume 2, Issue 1, 2021 
one-dimensional hyperbolic systems. The construction of ROMs for multidimensional hyperbolic systems remains an open challenge. We leave such multidimensional interpretations of the Koopman operator theory in terms of hodograph (or other) transforms for future studies. Another future work direction is to improve the current framework in handling experimental data, which are potentially contaminated by measurement noise. Due to the regularity at the shock front, we would expect Eulerian DMD approaches and regular DMD approaches to have stability problems. The current framework is expected to be more robust as the hodograph transform improves the regularity at the shock front (i.e., shocks become flat constants). For mixed wave problems, the current framework can still be sensitive to noise at the intersection of different waves. In an attempt to deal with experimental data, we would consider combining the proposed method with noise filters.

\section{ACKNOWLEDGMENTS}

This research was supported in part by Air Force Office of Scientific Research under award number FA9550-18-1-0474; by the Advanced Research Projects Agency-Energy (ARPA-E), U.S. Department of Energy, under Award Number DE-AR0001202; and by a gift from Total.

\section{REFERENCES}

Alessandro, A. and Nathan, K.J., Nonlinear Model Order Reduction via Dynamic Mode Decomposition, SIAM J. Sci. Comput., vol. 39, no. 5, pp. B778-B796, 2017.

Bear, J., Dynamics of Fluids in Porous Media, New York: Courier Corporation, 2013.

Benner, P., Gugercin, S., and Willcox, K., A Survey of Projection-Based Model Reduction Methods for Parametric Dynamical Systems, SIAM Rev., vol. 57, no. 4, pp. 483-531, 2015.

Brunton, S.L., Brunton, B.W., Proctor, J.L., and Kutz, J.N., Koopman Invariant Subspaces and Finite Linear Representations of Nonlinear Dynamical Systems for Control, PloS One, vol. 11, no. 2, p. e0150171, 2016.

Chang, T. and Hsiao, L., The Riemann Problem and Interaction of Waves in Gas Dynamics, Recon Tech. Report A 90, NASA STI, 1989.

Chaturantabut, S. and Sorensen, D.C., Nonlinear Model Reduction via Discrete Empirical Interpolation, SIAM J. Sci. Comput., vol. 32, no. 5, pp. 2737-2764, 2010.

Clarkson, P.A., Fokas, A.S., and Ablowitz, M.J., Hodograph Transformations of Linearizable Partial Differential Equations, SIAM J. Appl. Math., vol. 49, no. 4, pp. 1188-1209, 1989.

Courant, R. and Friedrichs, K.O., Supersonic Flow and Shock Waves, Vol. 21, Boston: Springer Science and Business Media, 1999.

Hamilton, W.R., The Hodograph, or a New Method of Expressing in Symbolic Language the Newtonian Law of Attraction, Proc. Roy. Irish Acad., vol. 3, pp. 344-353, 1847.

Harten, A., On the Symmetric Form of Systems of Conservation Laws with Entropy, J. Comput. Phys., vol. 49, pp. 151-164, 1983.

Kerschen, G., Golinval, J.C., Vakakis, A.F., and Bergman, L.A., The Method of Proper Orthogonal Decomposition for Dynamical Characterization and Order Reduction of Mechanical Systems: An Overview, Nonlinear Dyn., vol. 41, nos. 1-3, pp. 147-169, 2005.

Koopman, B.O., Hamiltonian Systems and Transformation in Hilbert Space, Proc. Natl. Acad. Sci. U.S.A., vol. 17, no. 5, p. 315, 1931.

Kutz, J.N., Brunton, S.L., Brunton, B.W., and Proctor, J.L., Dynamic Mode Decomposition: Data-Driven Modeling of Complex Systems, Philadelphia, PA: SIAM, 2016. 
Kutz, N.J., Proctor, J.L., and Brunton, S.L., Applied Koopman Theory for Partial Differential Equations and Data-Driven Modeling of Spatio-Temporal Systems, Complexity, vol. 2018, 2018.

Lax, P., Shock Waves and Entropy, Contributions to Nonlinear Functional Analysis, E.H. Zarantonello, Ed., New York: Elsevier, pp. 603-634, 1971.

LeVeque, R.J., Numerical Methods for Conservation Laws, Vol. 132, New York: Springer, 1992.

$\mathrm{Li}$, J. and Zheng, Y., Interaction of Rarefaction Waves of the Two-Dimensional Self-Similar Euler Equations, Arch. Rational Mech. Anal., vol. 193, no. 3, pp. 623-657, 2009.

Li, Q., Liu, J.G., and Shu, R., Sensitivity Analysis of Burger's Equation with Discontinuous Shocks, 2018. arXiv: 1708.04332

Lu, H. and Tartakovsky, D.M., Lagrangian Dynamic Mode Decomposition for Construction of Reduced-Order Models of Advection-Dominated Phenomena, J. Comput. Phys., vol. 407, p. 109229, 2020a.

Lu, H. and Tartakovsky, D.M., Prediction Accuracy of Dynamic Mode Decomposition, SIAM J. Sci. Comput., vol. 42, no. 3, pp. A1639-A1662, 2020b.

Majda, A. and Osher, S., Numerical Viscosity and the Entropy Condition, Commun. Pure Appl. Math., vol. 32, no. 6, pp. 797-838, 1979.

Mohyuddin, M.R., Siddiqui, A.M., Hayat, T., Siddiqui, J., and Asghar, S., Exact Solutions of TimeDependent Navier-Stokes Equations by Hodograph-Legendre Transformation Method, Tamsui Oxford J. Math. Sci., vol. 24, no. 3, pp. 257-268, 2008.

Mojgani, R. and Balajewicz, M., Lagrangian Basis Method for Dimensionality Reduction of Convection Dominated Nonlinear Flows, 2017. arXiv: 1701.04343

Morton, J., Jameson, A., Kochenderfer, M.J., and Witherden, F., Deep Dynamical Modeling and Control of Unsteady Fluid Flows, Advances in Neural Information Processing Systems (NIPS), S. Bengio, H. Wallach, H. Larochelle, K. Grauman, N. Cesa-Bianchi, and R. Garnett, Eds., vol. 31, pp. 9258-9268, 2018.

Oleinik, O.A., Discontinuous Solutions of Non-Linear Differential Equations, Uspekhi Matematicheskikh Nauk, vol. 12, no. 3, pp. 3-73, 1957.

Osher, S. and Chakravarthy, S., High Resolution Schemes and the Entropy Condition, SIAM J. Num. Anal., vol. 21, no. 5, pp. 955-984, 1984.

Rowley, C.W., Model Reduction for Fluids, Using Balanced Proper Orthogonal Decomposition, Int. J. Bifurc. Chaos, vol. 15, no. 03, pp. 997-1013, 2005.

Rowley, C.W., Mezić, I., Bagheri, S., Schlatter, P., and Henningson, D.S., Spectral Analysis of Nonlinear Flows, J. Fluid Mech., vol. 641, pp. 115-127, 2009.

Schmid, P.J., Dynamic Mode Decomposition of Numerical and Experimental Data, J. Fluid Mech., vol. 656, pp. 5-28, 2010.

Shu, F.H., The Physics of Astrophysics: Gas Dynamics, Vol. 2, New York: University Science Books, 1991.

Smoller, J., Shock Waves and Reaction-Diffusion Equations, vol. 258, Boston: Springer Science and Business Media, 2012.

Tu, J.H., Rowley, C.W., Luchtenburg, D.M., Brunton, S.L., and Kutz, J.N., On Dynamic Mode Decomposition: Theory and Applications, Comput. Dyn., vol. 1, pp. 391-421, 2014.

Whitham, G.B., Linear and Nonlinear Waves, vol. 42, New York: John Wiley and Sons, 2011.

Williams, M.O., Kevrekidis, I.G., and Rowley, C.W., A Data-Driven Approximation of the Koopman Operator: Extending Dynamic Mode Decomposition, J. Nonlin. Sci., vol. 25, no. 6, pp. 1307-1346, 2015.

Williams, M.O., Schmid, P.J., and Kutz, J.N., Hybrid Reduced-Order Integration with Proper Orthogonal Decomposition and Dynamic Mode Decomposition, Multiscale Model. Simul., vol. 11, no. 2, pp. 522544, 2013.

Volume 2, Issue 1, 2021 


\section{APPENDIX A. SCALAR CONSERVATION LAWS WITH CONVEX FLUXES}

Burgers' equation has a monotonically increasing flux function. Here, we extend our analysis to smooth, strictly convex flux functions $F(u)$. We consider a hyperbolic conservation law [Eq. (2.1)] defined for $(x, t) \in \mathbb{R} \times[0, T]$. It is subject to the initial condition $u(x, 0)=u_{0}(x)$, where the initial data $u_{0}(x)$ satisfy the following assumption.

Assumption A.1. The real-valued function $u_{0}(x)$ is such that

- $\lim _{x \rightarrow \pm \infty} u_{0}(x)=\mp 1$, and

- $u_{0}(x)$ is non-increasing and, therefore, the inverse function $x\left(u_{0}\right)$ is well defined on $-1 \leq$ $u_{0} \leq 1$.

Remark A.1. The domain of definition $x \in \mathbb{R}$ can be generalized to a finite-length interval $\left(u_{R}, u_{L}\right)$. The derivation is similar.

\section{A.1 Solution before Shock Formation}

Similar to Section 3.1, hodograph transformation yields an equation for $x(t, u)$ :

$$
\frac{\mathrm{d} x}{\mathrm{~d} t}(t, u)=f(u), \quad x(0, u)=x_{0}(u) ; \quad u \in(-1,1) .
$$

The convexity of $F(u)$ ensures that its derivative $f(u)$ is an increasing function. Let $G$ denote the inverse function of $f$ :

$$
G[f(u)]=f[G(u)]=u .
$$

Then, defining $y(t, u)=x[t, G(u)]$, Eq. (A.1) becomes

$$
\frac{\mathrm{d} y}{\mathrm{~d} t}(t, u)=u, \quad y(0, u)=y_{0}(u)=x_{0}[G(u)] ; \quad u \in(-1,1) .
$$

Differentiating both sides of this equation with respect to $u$,

$$
\frac{\partial^{2} y}{\partial t \partial u}=1
$$

which gives

$$
\frac{\mathrm{d} u}{\mathrm{~d} t}(t, u)=y_{0}^{\prime}(u)+t
$$

Therefore the shock formation time is determined by

$$
t^{*}=-\min _{u} y_{0}^{\prime}(u)=-y_{0}^{\prime}\left[f\left(u^{*}\right)\right]
$$

\section{A.2 Solution after Shock Formation}

The shock speed $s$ is given by the Rankine-Hugoniot condition,

$$
s=\frac{F\left(u_{1}\right)-F\left(u_{2}\right)}{u_{1}-u_{2}},
$$


where $u_{1}(t)$ and $u_{2}(t)$ are defined as the limits of $u(t)$ from the top and bottom of the shock, respectively. Since $s=\mathrm{d} x^{*} / \mathrm{d} t$, this gives an equation for the shock trajectory $x^{*}(t)$,

$$
\frac{\mathrm{d} x^{*}}{\mathrm{~d} t}=\frac{F\left(u_{1}\right)-F\left(u_{2}\right)}{u_{1}-u_{2}} .
$$

A system of coupled ODEs for $u_{1}(t)$ and $u_{2}(t)$ is derived in Li et al. (2018):

$$
\begin{aligned}
\frac{\mathrm{d} u_{1}}{\mathrm{~d} t} & =F_{1}\left(u_{1}, u_{2}\right) \equiv \frac{1}{g\left(u_{1}\right)-f^{\prime}\left(u_{1}\right) t}\left[f\left(u_{1}\right)-\frac{F\left(u_{1}\right)-F\left(u_{2}\right)}{u_{1}-u_{2}}\right], \\
\frac{\mathrm{d} u_{2}}{\mathrm{~d} t} & =F_{2}\left(u_{1}, u_{2}\right) \equiv \frac{1}{g\left(u_{2}\right)-f^{\prime}\left(u_{2}\right) t}\left[f\left(u_{2}\right)-\frac{F\left(u_{1}\right)-F\left(u_{2}\right)}{u_{1}-u_{2}}\right],
\end{aligned}
$$

where $g(u)=-x_{0}^{\prime}(u)$. These ODEs are subject to initial conditions $u_{1}\left(t^{*}\right)=u^{*}$ and $u_{2}\left(t^{*}\right)=$ $u^{*}$.

\section{A.3 Summary of Hodograph Solution}

In summary, the reformulation for general scalar conservation law with convex flux is

$$
\begin{cases}t<t^{*}: & \text { Eq. (A.1), } \\ t>t^{*}: & \begin{cases}\text { Eq. (A.1) for } u \in\left(u_{R}, u_{2}\right) \cup\left(u_{1}, u_{L}\right), \\ \text { Eq. (A.8) for } u \in\left(u_{2}, u_{1}\right)\end{cases} \end{cases}
$$

where $t^{*}=-x_{0}^{\prime}\left(u^{*}\right)$.

Remark A.2. We can show that $u_{1}(t)$ is monotonically increasing in time and $u_{2}(t)$ is monotonically decreasing, so that

$$
u_{1} \geq u^{*}, \quad u_{2} \leq u^{*}, \quad x_{0}^{\prime}\left(u_{1}\right)+t \leq 0, \quad x_{0}^{\prime}\left(u_{2}\right)+t \leq 0 .
$$

In many cases of interests, and in our numerical experiment, either $u_{2}=u_{R}$ and $u_{1}=u_{L}$ or $\left|u_{2}-u_{1}\right| \ll \Delta t$ (so that $u_{2} \approx u_{R}$ and $u_{1} \approx u_{L}$ ). This allows us to focus on shock propagation [i.e., on Eq. (3.7)] without having to solve Eq. (A.9).

Remark A.3. For the more general initial condition $u_{0}$, we need to decompose $u_{0}(x)$ into regions of monotonicity. Each monotonic piece of $u_{0}$ would have a unique inverse function $x_{0}\left(u_{0}\right)$. Then, based on the generalized entropy condition, we construct the convex hull for the flux function $F(u)$, providing a way to decompose the initial data. Shock propagating initial data and rarefaction propagating initial data are determined afterward. Then, the full solution is the combination of the rarefaction pieces and the shock pieces.

Volume 2, Issue 1, 2021 